MATHEMATICS OF COMPUTATION

Volume 77, Number 262, April 2008, Pages 909-941

S 0025-5718(07)02064-9

Article electronically published on November 16, 2007

\title{
COUPLING OF GENERAL LAGRANGIAN SYSTEMS
}

\author{
A. AmBroso, C. CHALONS, F. COQUEL, E. GODLEWSKI, F. LAGOUTIÈRE, \\ P.-A. RAVIART, AND N. SEGUIN
}

\begin{abstract}
This work is devoted to the coupling of two fluid models, such as two Euler systems in Lagrangian coordinates, at a fixed interface. We define coupling conditions which can be expressed in terms of continuity of some well chosen variables and then solve the coupled Riemann problem. In the present setting where the interface is characteristic, a particular choice of dependent variables which are transmitted ensures the overall conservativity.
\end{abstract}

\section{INTRODUCTION}

The problem of coupling two different fluid models at a fixed interface stems from the need of coupling thermal-hydraulic models within the frame of a new generation of two-phase flow codes for nuclear reactors. These codes are generally built upon distinct models, each being devoted to the particular flow conditions taking place in a given reactor component. The simulation of the whole device thus requires transient exchange of information at the interface of two adjacent components. Let us emphasize that the coupling problem actually arises in various other physical settings (see 13 for instance).

In ideal cases, physical arguments, such as the continuity of some primitive quantity, might help in defining the transmission or coupling conditions. Even in this case, both theoretical considerations and numerical results obtained on some significant tests when coupling Euler systems (see 3]) will prove that not any coupling based on continuity arguments is feasible. This also gives rise to interesting questions (nonuniqueness of self-similar solutions) and has led us to analyze the problem in an abstract frame.

The theoretical study of these coupling conditions was initiated in the scalar case [12, and then for linear systems and the usual Lagrangian system in [13]. In the first paper new coupling conditions have been formalized which result by expressing that two boundary value problems should be well-posed, and resume to impose (whenever possible) the continuity of the solution at the interface without imposing the overall conservativity of the coupled model. For hyperbolic systems of conservation laws, the well-posedness of initial boundary value problems is difficult and the boundary conditions have been expressed in terms of Riemann problems in 13. This approach is well suited for the numerical methods that we are interested in implementing and is linked to the theoretical results concerning the convergence

Received by the editor July 26, 2006 .

2000 Mathematics Subject Classification. Primary 35L50, 35L65, 76M12, 76N15.

Key words and phrases. Hyperbolic systems, coupling, Riemann problems, finite volume methods, fluid model.

(C)2007 American Mathematical Society Reverts to public domain 28 years from publication 
of the 'two-flux method' in the scalar case (see [12] and also [10]). In fact this formalism can be well understood in the particular case of the Euler system in Lagrangian coordinates since the geometry of characteristics at the interface is fixed and no resonance phenomenon can occur. This enables us to express the coupling conditions in terms of continuity of some variables and then to solve the coupled Riemann problem in a unique way. In this work we justify the choice of dependent variables which are transmitted.

This may seem at first glance a rather theoretical exemple. On the one hand, it is indeed a very particular and very interesting case of coupling to analyze because of the special property that 0 is an eigenvalue. On the other hand, the analysis will justify the use of 'Lagrange+projection' schemes when coupling systems in Eulerian coordinates at a fixed interface (cf. 6]), which means that it provides a useful tool to couple two Euler systems at a fixed interface (which in that case is not characteristic). One may then ask why couple two fluid models with different equations of state at a fixed interface since it may seem an unphysical example. The answer is that it is a simplified model of what we get when coupling more complex models associated to different systems of pde's whose closure laws are not strictly compatible, as will happen for instance in the context of thermal-hydraulic models. The main lines of the present work were announced in [5].

The outline of the paper is as follows. In Section 2, we introduce the framework of interface coupling and define the coupling conditions in terms of traces of solutions of Riemann problems. Then in Section 3 these conditions are explicit and the coupled Riemann problem is solved for two $p$-systems in the case of two Euler systems in Lagrangian coordinates. Section 4 treats the coupling of two systems of different dimensions: the $p$-system and the Euler system. Section 5 introduces a more general theoretical setting following Després' formalism (cf. 7]) in order to extend the coupling to more general Lagrangian systems. Some changes of variables are introduced in order to express the coupling conditions and the coupled Riemann problem is solved. Several numerical results will illustrate the theory.

\section{THE INTERFACE COUPLING APPROACH}

2.1. The coupling procedure. We first describe the theoretical settings and make precise our notation.

Let $\Omega \subset \mathbb{R}^{q}$ be the set of states and let $\mathbf{f}_{\alpha}, \alpha=L, R$ be two smooth functions from $\Omega$ into $\mathbb{R}^{q}$. Given a function $\mathbf{u}_{0}: x \in \mathbb{R} \rightarrow \mathbf{u}_{0}(x)$, we want to find a function $\mathbf{u}:(x, t) \in \mathbb{R} \times \mathbb{R}_{+} \rightarrow \mathbf{u}(x, t) \in \Omega$ solution of

$$
\begin{aligned}
\partial_{t} \mathbf{u}+\partial_{x} \mathbf{f}_{L}(\mathbf{u})=\mathbf{0}, & x<0, t>0, \\
\partial_{t} \mathbf{u}+\partial_{x} \mathbf{f}_{R}(\mathbf{u})=\mathbf{0}, & x>0, t>0,
\end{aligned}
$$

satisfying the initial condition

$$
\mathbf{u}(x, 0)=\mathbf{u}_{0}(x), \quad x \in \mathbb{R},
$$

and at the interface $x=0$, a coupling condition which we now describe.

This coupling condition has been chosen in order to obtain two well-posed initial boundary value problems in $x>0, t \geq 0$ and in $x<0, t \geq 0$. This means that the trace $\mathbf{u}(0-, t)$ (resp. $\mathbf{u}(0+, t)$ ) should be an admissible boundary condition at $x=0$ for the system in $x>0, t \geq 0$ (resp. $\mathbf{u}(0+, t)$ is an admissible boundary condition at $x=0$ for the system in $x<0, t \geq 0)$. We will assume that the 
systems are hyperbolic, i.e. for $\alpha=L, R$, the Jacobian matrix $A_{\alpha}(\mathbf{u}) \equiv \mathbf{f}_{\alpha}^{\prime}(\mathbf{u})$ of $\mathbf{f}_{\alpha}(\mathbf{u})$ is diagonalizable with real eigenvalues $\lambda_{\alpha, k}(\mathbf{u})$ and corresponding eigenvectors $\mathbf{r}_{\alpha, k}(\mathbf{u}), 1 \leq k \leq q$. Then rigorous ways of writing the boundary conditions can be found in [9], 14] but the most practical way to express them involves the traces of the solution of a Riemann problem. Thus, we introduce the self-similar solution

$$
\mathbf{u}(x, t)=\mathbf{W}_{\alpha}\left(x / t ; \mathbf{u}_{L}, \mathbf{u}_{R}\right)
$$

of the Riemann problem for the system associated to the flux $\mathbf{f}_{\alpha}$, i.e. the Cauchy problem with initial condition

$$
\mathbf{u}(x, 0)= \begin{cases}\mathbf{u}_{L}, & x<0 \\ \mathbf{u}_{R}, & x>0\end{cases}
$$

We set for all $\mathbf{b} \in \Omega$,

$$
\begin{aligned}
& \mathcal{O}_{L}(\mathbf{b})=\left\{\mathbf{w}=\mathbf{W}_{L}\left(0-; \mathbf{u}_{\ell}, \mathbf{b}\right) ; \mathbf{u}_{\ell} \in \Omega\right\} \\
& \mathcal{O}_{R}(\mathbf{b})=\left\{\mathbf{w}=\mathbf{W}_{R}\left(0+; \mathbf{b}, \mathbf{u}_{r}\right) ; \mathbf{u}_{r} \in \Omega\right\}
\end{aligned}
$$

and following $[8$ we define admissible boundary conditions of the form

$$
\mathbf{u}(0-, t) \in \mathcal{O}_{L}(\mathbf{b}(t)), \quad t>0,
$$

and

$$
\mathbf{u}(0+, t) \in \mathcal{O}_{R}(\mathbf{b}(t)), \quad t>0,
$$

for (1) and (2), respectively. Hence natural coupling conditions for problems (1)-(2) consist in requiring

$$
\left\{\begin{array}{l}
\mathbf{u}(0-, t) \in \mathcal{O}_{L}(\mathbf{u}(0+, t)) \\
\mathbf{u}(0+, t) \in \mathcal{O}_{R}(\mathbf{u}(0-, t)) .
\end{array}\right.
$$

This means that at each time $t>0$, there exist some states $\mathbf{u}_{-}(t), \mathbf{u}_{+}(t) \in \Omega$ such that $\mathbf{u}(0-, t)=\mathbf{W}_{L}\left(0-; \mathbf{u}_{-}(t), \mathbf{u}(0+, t)\right)$ and $\mathbf{u}(0+, t)=\mathbf{W}_{R}\left(0+; \mathbf{u}(0-, t), \mathbf{u}_{+}(t)\right)$. Using the formulation with Riemann problems to express admissible boundary conditions is more practical and suitable for the numerical approximation of the coupled problem. It is thoroughly justified in the scalar case and for linear systems. In [12, devoted to the scalar case, we have shown that this was indeed a reasonable way of coupling two scalar conservation laws in the sense that, in meaningful situations, the coupled problem has a unique solution and a 'natural' numerical upwind scheme (the so-called two-flux scheme) converges to this solution. The case of linear systems is treated in [13].

Condition (5) resumes in a number of cases to the continuity of the solution at the interface

$$
\mathbf{u}(0-, t)=\mathbf{u}(0+, t)
$$

at least (6) holds true whenever the interface is noncharacteristic. Thus we may interpret the coupling condition as a way of ensuring in a weak sense the continuity or the transmission of the conservative variables $\mathbf{u}$.

However, when dealing with physical systems, we may prefer to transmit not the conservative variables but other physical variables. Indeed, define two distinct changes of variables

$$
\mathbf{v} \rightarrow \mathbf{u}=\varphi_{\alpha}(\mathbf{v}), \alpha=L, R
$$


from some set $\Omega_{\mathbf{v}} \subset \mathbb{R}^{q}$ onto $\Omega$ such that $\varphi_{\alpha}^{\prime}(\mathbf{v})$ is an isomorphism of $\mathbb{R}^{q}$. Then if $\mathbf{c}$ is a given boundary physical data, setting $\mathbf{b}_{\alpha}=\varphi_{\alpha}(\mathbf{c})$, we define

$$
\begin{aligned}
& \mathcal{O}_{L}\left(\mathbf{b}_{L}\right)=\left\{\mathbf{w}=\mathbf{W}_{L}\left(0-; \mathbf{u}_{\ell}, \mathbf{b}_{L}\right) ; \mathbf{u}_{\ell} \in \Omega\right\}, \\
& \mathcal{O}_{R}\left(\mathbf{b}_{R}\right)=\left\{\mathbf{w}=\mathbf{W}_{R}\left(0+; \mathbf{b}_{R}, \mathbf{u}_{r}\right) ; \mathbf{u}_{r} \in \Omega\right\}
\end{aligned}
$$

which are admissible boundary conditions for the systems (11) and (2), respectively. Thus we now require

$$
\left\{\begin{array}{l}
\mathbf{u}(0-, t) \in \mathcal{O}_{L}\left(\varphi_{L}(\mathbf{v}(0+, t))\right), \\
\mathbf{u}(0+, t) \in \mathcal{O}_{R}\left(\varphi_{R}(\mathbf{v}(0-, t))\right) .
\end{array}\right.
$$

Since $\varphi_{L}(\mathbf{v}(0+, t)) \neq \varphi_{R}(\mathbf{v}(0+, t))=\mathbf{u}(0+, t)$, the boundary sets in (77) and (15) are distinct. Conditions (7) will ensure the transmission of physical variables and whenever possible their continuity instead of (6)

$$
\mathbf{v}(0-, t)=\mathbf{v}(0+, t)
$$

again when the interface is noncharacteristic.

Next we will be interested in solving the coupled Riemann problem (1), (22), (3) with coupling conditions given either by (5) or by (7) for some change of variables $\varphi_{\alpha}$ to be specified.

2.2. Numerical coupling. Let us briefly recall the numerical procedure for the sake of completeness since numerical illustrations are provided in the following sections. We use a finite volume method for the discretization of each system (11), (2). Let $\Delta x, \Delta t$, denote the uniform space and time steps, set $\mu=\Delta t / \Delta x$, $t_{n}=n \Delta t, n \in \mathrm{N}$, and consider the cells $C_{j+1 / 2}=\left(x_{j}, x_{j+1}\right)$, with center $x_{j+1 / 2}=$ $(j+1 / 2) \Delta x, j \in \mathrm{Z}$. The initial condition is discretized as usual by

$$
\mathbf{u}_{j+1 / 2}^{0}=\frac{1}{\Delta x} \int_{C_{j+1 / 2}} \mathbf{u}_{0}(x) d x, j \in \mathrm{Z} .
$$

For the numerical coupling, we are given two numerical fluxes $\mathbf{g}_{L}, \mathbf{g}_{R}$ ( $\mathbf{g}_{\alpha}$ is consistent with $\mathbf{f}_{\alpha}$ ) corresponding to 3-point schemes (we assume these schemes are monotone in the scalar case under some CFL condition). We define the scheme by

$$
\begin{gathered}
\mathbf{u}_{j-1 / 2}^{n+1}=\mathbf{u}_{j-1 / 2}^{n}-\mu\left(\mathbf{g}_{L, j}^{n}-\mathbf{g}_{L, j-1}^{n}\right), \quad j \leq 0, n \geq 0, \\
\mathbf{u}_{j+1 / 2}^{n+1}=\mathbf{u}_{j+1 / 2}^{n}-\mu\left(\mathbf{g}_{R, j+1}^{n}-\mathbf{g}_{R, j}^{n}\right), \quad j \geq 0, n \geq 0
\end{gathered}
$$

(see also 1 in another context). So we have one fixed interface at $x=0$ and two fluxes $\mathbf{g}_{\alpha, 0}^{n}$. We have $\mathbf{g}_{\alpha, j}^{n}=\mathbf{g}_{\alpha}\left(\mathbf{u}_{j-1 / 2}^{n}, \mathbf{u}_{j+1 / 2}^{n}\right), \alpha=L, j<0, \alpha=R, j>0$, and for the fluxes at the interface $x=0$, we choose $\mathbf{g}_{\alpha, 0}^{n}$ according to the coupling procedure. The choice

$$
\mathbf{g}_{\alpha, 0}^{n}=\mathbf{g}_{\alpha}\left(\mathbf{u}_{-1 / 2}^{n}, \mathbf{u}_{1 / 2}^{n}\right), \alpha=L, R,
$$

corresponds to transmit the conservative variables $\mathbf{u}$. Namely, if $j \geq 0$, the scheme (10) with flux $\mathbf{g}_{R}$ consistent with $\mathbf{f}_{R}$ approximates the IBVP (2) with initial condition $u(x, 0)=u_{0}(x), x>0$, and for boundary condition at $x=0$, the scheme takes $\mathbf{u}_{-1 / 2}^{n}$. Since $\mathbf{g}_{L, 0}^{n} \neq \mathbf{g}_{R, 0}^{n}$, it is a nonconservative numerical approach, as for the continuous problem. For example, the flux at the boundary with Godunov's scheme is $\mathbf{g}_{R, 0}^{n}=\mathbf{f}_{R}\left(\mathbf{W}_{R}\left(0+; \mathbf{u}_{-1 / 2}^{n}, \mathbf{u}_{1 / 2}^{n}\right)\right)$. It has been proved in the scalar case (cf. [12]) that in a number of practical situations, scheme (9)-(10) converges towards a solution of (11)-(2) satisfying (5). 


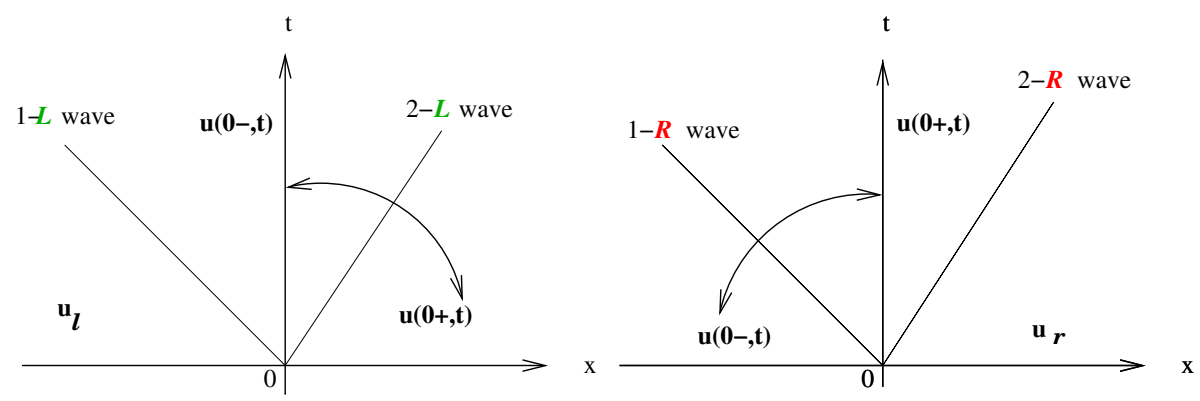

FiguRE 1. The coupling conditions (5) for the $p$-system

We can also transmit the physical variables $\mathbf{v}$ by choosing

$$
\mathbf{g}_{L, 0}^{n}=\mathbf{g}_{L}\left(\mathbf{u}_{-1 / 2}^{n}, \varphi_{L}\left(\mathbf{v}_{1 / 2}^{n}\right)\right), \mathbf{g}_{R, 0}^{n}=\mathbf{g}_{R}\left(\varphi_{R}\left(\mathbf{v}_{-1 / 2}^{n}\right), \mathbf{u}_{1 / 2}^{n}\right)
$$

where $\mathbf{v}_{1 / 2}^{n}=\varphi_{R}^{-1}\left(\mathbf{u}_{1 / 2}^{n}\right), \mathbf{v}_{-1 / 2}^{n}=\varphi_{L}^{-1}\left(\mathbf{u}_{-1 / 2}^{n}\right)$.

2.3. A canonical example: coupling two $p$-systems. We are going to illustrate the two choices in the coupling procedure on the $p$-system:

$$
\left\{\begin{array}{l}
\partial_{t} \tau-\partial_{x} v=0 \\
\partial_{t} v+\partial_{x} p=0
\end{array}\right.
$$

and then, in the following sections, for the Euler system in Lagrangian coordinates. Note however that in this latter case, the interface is characteristic and will correspond to a contact discontinuity. Hence in general, the coupling does not yield the continuity (6) or (8); we will see that it yields the continuity of a subset of variables.

In (11), $x$ stands for a mass variable, $\tau$ denotes the specific volume, $v$ the velocity, and we assume that the pressure $p$ is a given decreasing function $p=p(\tau)$. Let us consider the coupling of two $p$-systems

$$
\partial_{t} \mathbf{u}+\partial_{x} \mathbf{f}_{\alpha}(\mathbf{u})=\mathbf{0}, \alpha=L \text { in } x<0, \alpha=R \text { in } x>0,
$$

where

$$
\left\{\begin{array}{l}
\mathbf{u}=(\tau, v)^{T}, \tau>0 \\
\mathbf{f}_{L}(\mathbf{u})=(-v, p)^{T}, p=p_{L}(\tau) \\
\mathbf{f}_{R}(\mathbf{u})=(-v, p)^{T}, p=p_{R}(\tau) .
\end{array}\right.
$$

We assume that $p_{\alpha}^{\prime}<0, p_{\alpha}^{\prime \prime}>0, \alpha=L, R$. The two systems differ by the pressure law $p$. An important feature is that the signs of the two eigenvalues do not depend on $\mathbf{u}: \lambda_{1}(\mathbf{u})=-c<0<\lambda_{2}(\mathbf{u})=c, c=\sqrt{-p^{\prime}(\tau)}$. Hence in the left (resp. right) half plane, there can be only 1 -waves (resp. 2-waves).

We first transmit the conservative variables $(\tau, v)$. We denote by $\mathscr{C}_{\alpha}^{k}\left(\mathbf{u}_{-}\right)$the $k \alpha$-wave curve, i.e. the set of states that can be connected to a given state $\mathbf{u}_{-}$ by a $k \alpha$-wave, $k=1,2$ (either rarefaction or admissible shock), relative to the $p$-system with flux $\mathbf{f}_{\alpha}, \alpha=L, R$. Expressing the coupling conditions (5) gives (for the left condition $\left.\mathbf{u}(0-, t) \in \mathcal{O}_{L}(\mathbf{u}(0+, t))\right)$ that $\mathbf{u}(0-, t)$ is connected to $\mathbf{u}(0+, t)$ by a $2 L$-wave which means $\mathbf{u}(0+, t) \in \mathscr{C}_{L}^{2}(\mathbf{u}(0-))$ (we use shortened notation for $\mathscr{C}_{L}^{2}(\mathbf{u}(0-, t)$ ), and similarly (for the right condition) by a $1 R$-wave (see Figure 1). Thus $\mathbf{u}(0+, t) \in \mathscr{C}_{L}^{2}(\mathbf{u}(0-)) \cap \mathscr{C}_{R}^{1}(\mathbf{u}(0-))$ and, since it is well known that the 
two wave curves intersect at only one point in the plane $(\tau, v)$, at least away from vacuum (see for instance [11]), it yields

$$
\mathbf{u}(0+, t)=\mathbf{u}(0-, t) .
$$

Now the IBVP's in both quarter planes $(x<0, t>0)$ and $(x>0, t>0)$ are also well posed if one wishes to prescribe a given $(v, p)$ on $x=0$ in a weak sense, according to (7). Indeed, by assumption $p_{\alpha}^{\prime}<0$, hence, we can define its inverse mapping $\tau_{\alpha}(p)$ for $\alpha=L, R$. Setting $\mathbf{v}=(v, p)^{T}$, we have an admissible change of variables: $\mathbf{u}=\varphi_{\alpha}(\mathbf{v})$ where

$$
(v, p) \rightarrow \varphi_{\alpha}(v, p) \equiv(\tau, v)
$$

is simply defined by $\tau=\tau_{\alpha}(p)$ (for instance if $p_{\alpha}(\tau)=\tau^{-\gamma_{\alpha}}$, then $\tau_{\alpha}(p)=p^{-1 / \gamma_{\alpha}}$ ).

We now transmit this set of variables $(v, p)$.

Proposition 1. For the systems (13), the coupling conditions (7) are equivalent to

$$
\left\{\begin{array}{l}
v(0-, t)=v(0+, t), \\
p(0-, t)=p(0+, t) .
\end{array}\right.
$$

Moreover, the solution of the coupled Riemann problem (12), (13), (3), (17) exists and is unique.

Proof. Let us express the coupling condition (7): $\mathbf{u}(0+, t) \in \mathcal{O}_{R}\left(\varphi_{R}(\mathbf{v}(0-, t))\right)$ and $\mathbf{u}(0-, t) \in \mathcal{O}_{L}\left(\varphi_{L}(\mathbf{v}(0+, t))\right)$, with more precisely $\mathbf{v}(0 \pm, t)=(v(0 \pm), p(0 \pm, t))^{T}$ and $\mathbf{u}(0-, t)=\varphi_{L}(\mathbf{v}(0-, t)), \mathbf{u}(0+, t)=\varphi_{R}(\mathbf{v}(0+, t))$.

First $\mathbf{u}(0+, t) \in \mathcal{O}_{R}\left(\varphi_{R}(\mathbf{v}(0-, t))\right.$ yields that $\varphi_{R}(\mathbf{v}(0-, t))$ is connected to $\mathbf{u}(0+, t)$ $=\varphi_{R}(\mathbf{v}(0+, t))$ by a $1 R$-wave. The idea is that we can parametrize the wave curves by $p$ and represent them in the $(v, p)$-plane (for details concerning the equations of the wave-curves, see [11], Chapter I, section 7$)$. If the $1 R$-wave curve is $\mathscr{C}_{R}^{1}(\mathbf{u}(0-))=\left\{(\tau, v) ; v=\Psi_{1, R}(\tau)\right\}$, let

$$
\begin{aligned}
\tilde{\mathcal{C}}_{R}^{1}(\mathbf{v}(0-)) & =\left\{(v, p) ; v=\Psi_{1, R}\left(\tau_{R}(p)\right)\right\}=\left\{(v, p) ; \varphi_{R}(v, p) \in \mathscr{C}_{R}^{1}(\mathbf{u}(0-))\right\} \\
& =\varphi_{R}^{-1}\left(\mathscr{C}_{R}^{1}(\mathbf{u}(0-))\right)
\end{aligned}
$$

be its representation in the $(v, p)$-coordinates. We then have $\mathbf{v}(0+, t) \in \tilde{\mathcal{C}}_{R}^{1}(\mathbf{v}(0-))$ (see Figure 2).

Similarly, $\mathbf{u}(0-, t) \in \mathcal{O}_{L}\left(\varphi_{L}(\mathbf{v}(0+, t))\right)$ yields that $\mathbf{u}(0-, t)=\varphi_{L}(\mathbf{v}(0-, t))$ is connected to $\varphi_{L}(\mathbf{v}(0+, t))$ by a $2 L$-wave. We parametrize the wave curves by $p$ and represent them onto the $(v, p)$-plane. If the $2 L$-wave curve is $\mathscr{C}_{L}^{2}(\mathbf{u}(0-))=$ $\left\{(\tau, v) ; v=\Psi_{2, L}(\tau)\right\}$, let

$$
\begin{aligned}
\tilde{\mathcal{C}}_{L}^{2}(\mathbf{v}(0-)) & =\left\{(v, p) ; v=\Psi_{2, L}\left(\tau_{L}(p)\right)\right\}=\left\{(v, p) ; \varphi_{L}(v, p) \in \mathscr{C}_{L}^{2}(\mathbf{u}(0-))\right\} \\
& =\varphi_{L}^{-1}\left(\mathscr{C}_{L}^{2}(\mathbf{u}(0-))\right)
\end{aligned}
$$

be its representation in the $(v, p)$-coordinates. We then have $\mathbf{v}(0+, t) \in \tilde{\mathcal{C}}_{L}^{2}(\mathbf{v}(0-))$.

We have $\mathbf{v}(0+, t) \in \tilde{\mathcal{C}}_{R}^{1}(\mathbf{v}(0-)) \cap \tilde{\mathcal{C}}_{L}^{2}(\mathbf{v}(0-))$. Thus $\mathbf{v}(0+, t)=\mathbf{v}(0-, t)$ because it is easy to prove that the two curves intersect at only one point in the plane $(v, p)$. Hence we do have continuity of $v, p$, not of $\tau$ since $\tau(0+, t)=p(0+, t)^{-1 / \gamma_{R}} \neq$ $p(0-, t)^{-1 / \gamma_{L}}=\tau(0-, t)$. 

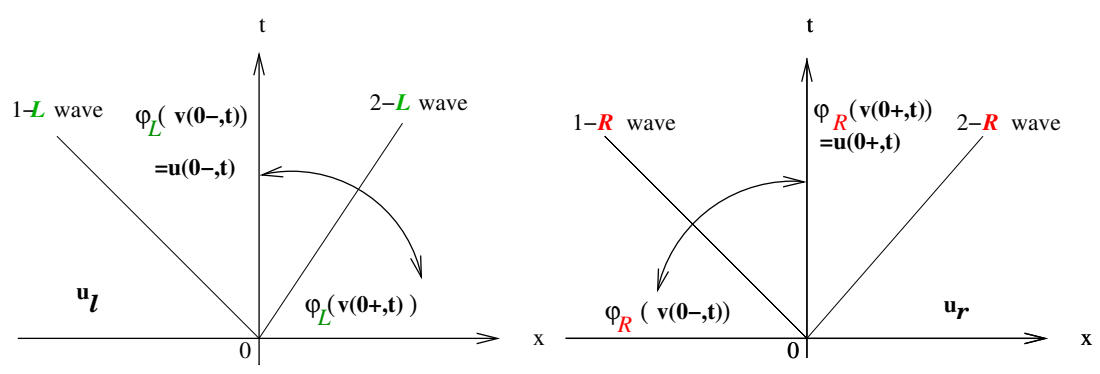

FiguRE 2. The coupling conditions (7) for the $p$-system

Under the assumptions made on the $p_{\alpha}$ 's, existence and uniqueness of the solution of the coupled Riemann problem, away from vacuum, follow as in the usual noncoupled case.

The solution of a coupled Riemann problem is illustrated in Figure 3 for two $\gamma$-laws, $p_{\alpha}(\tau)=\tau^{-\gamma_{\alpha}}, \gamma_{L}=1.4, \gamma_{R}=1.6$.

Remark 1 . The choice of the transmitted variables is clearly nonunique. The above argument may be used for any other admissible change of variables of the form $\mathbf{u}=(\tau, v) \rightarrow \mathbf{v}=\left(v, h_{\alpha}(\tau)\right)$ provided the corresponding functions $h_{\alpha}, \alpha=L, R$, are both strictly increasing or both strictly decreasing mappings. It happens that the flux $(-v, p)$ in the preceding example may be taken as a set of dependent variables. It will not be possible in the next example, where the flux is $(-v, p, p v)$, since it would not define an admissible change of variables. However, we may want to transmit part of the flux variables $(v, p)$ which will then also yield the transmission of $v p$. Hence, in these particular examples, we are able to couple the two models by imposing the continuity of the flux, which we call flux coupling and which might appear as a conservative approach.

\section{Coupling two Euler systems in Lagrangian Coordinates}

3.1. The Euler system. We consider the full Euler system of gas dynamics in Lagrangian coordinates

$$
\partial_{t} \mathbf{u}+\partial_{x} \mathbf{f}(\mathbf{u})=\mathbf{0}
$$

where

$$
\mathbf{u}=(\tau, v, e)^{T}, \mathbf{f}(\mathbf{u})=(-v, p, p v)^{T} .
$$

In (17), $x$ stands for a mass variable, while in (18), $\tau$ denotes the specific volume, $v$ the velocity, $e=\varepsilon+\frac{1}{2} v^{2}$ the specific total energy, and $\varepsilon$ the specific internal energy. We assume that the pressure $p$ is a given function $p=p(\tau, \varepsilon)$. We study the coupling of two such systems at the interface $x=0$ which now happens to be characteristic. It thus has the physical interpretation of a contact discontinuity separating two fluids with different equations of state

$$
p=p_{\alpha}(\tau, \varepsilon), \alpha=L, R \text {. }
$$

We denote by

$$
\mathbf{f}_{\alpha}(\mathbf{u})=(-v, p, p v)^{T}, p=p_{\alpha}(\tau, \varepsilon), \alpha=L, R,
$$

the corresponding flux functions. 

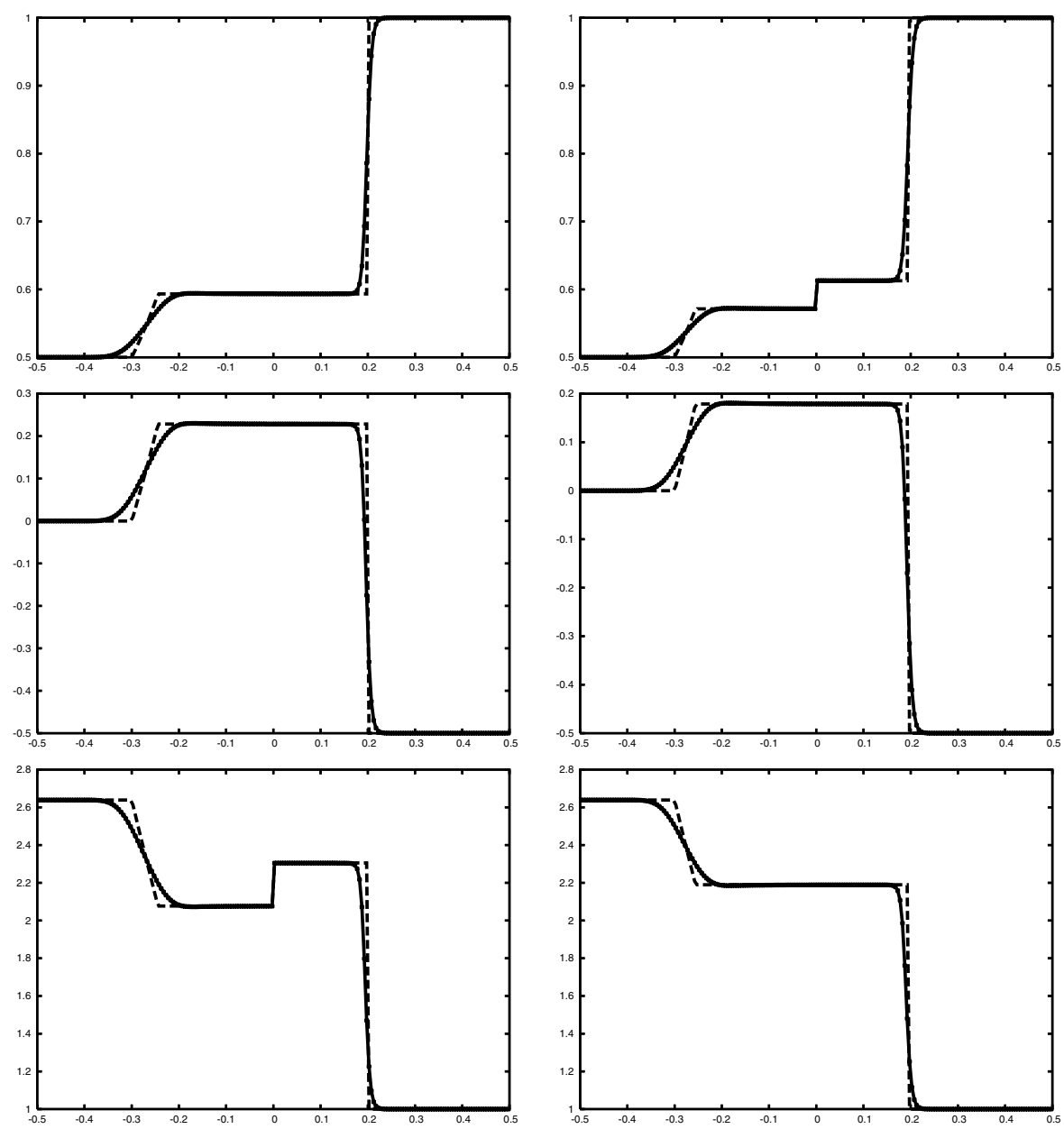

Figure 3. $\tau, v, p$ in the transmission of $\mathbf{u}=(\tau, v)$ left / vs. $\mathbf{v}=$ $(v, p)$ right for the coupled $p$-system: continuity of $(\tau, v)$ left / vs. continuity of $(v, p)$ right (numerical and exact solution)

Let $\mathbf{A}(\mathbf{u})$, be the Jacobian matrix of $\mathbf{f}(\mathbf{u})$

$$
\mathbf{A}(\mathbf{u})=\left(\begin{array}{ccc}
0 & -1 & 0 \\
p_{\tau} & -v p_{\varepsilon} & p_{\varepsilon} \\
v p_{\tau} & p-v^{2} p_{\varepsilon} & v p_{\varepsilon}
\end{array}\right)
$$

with the notations

$$
p_{\varepsilon}=\frac{\partial p}{\partial \varepsilon}(\tau, \varepsilon), p_{\tau}=\frac{\partial p}{\partial \tau}(\tau, \varepsilon),
$$

and we note when necessary by $\mathbf{A}_{\alpha}(\mathbf{u})$ that of $\mathbf{f}_{\alpha}(\mathbf{u}), \alpha=L, R$. The eigenvalues of $\mathbf{A}(\mathbf{u})$ are

$$
\lambda_{1}(\mathbf{u})=-C(\mathbf{u})<\lambda_{2}=0<\lambda_{3}(\mathbf{u})=C(\mathbf{u}),
$$

where

$$
C(\mathbf{u})=\sqrt{-p_{\tau}+p p_{\varepsilon}}
$$


denotes the Lagrangian sound speed. Recall that the right eigenvectors of $\mathbf{A}(\mathbf{u})$ can be chosen as

$$
\mathbf{r}_{1}(\mathbf{u})=\left(\begin{array}{c}
-1 \\
-C \\
p-C v
\end{array}\right), \mathbf{r}_{2}(\mathbf{u})=\left(\begin{array}{c}
p_{\varepsilon} \\
0 \\
-p_{\tau}
\end{array}\right), \mathbf{r}_{3}(\mathbf{u})=\left(\begin{array}{c}
-1 \\
C \\
p+C v
\end{array}\right),
$$

while the left eigenvectors are given by

$$
\mathbf{l}_{1}(\mathbf{u})=\frac{1}{2 C^{2}}\left(\begin{array}{c}
p_{\tau} \\
-C-v p_{\varepsilon} \\
p_{\varepsilon}
\end{array}\right), \mathbf{l}_{2}(\mathbf{u})=\frac{1}{C^{2}}\left(\begin{array}{c}
p \\
-v \\
1
\end{array}\right), \mathbf{l}_{3}(\mathbf{u})=\frac{1}{2 C^{2}}\left(\begin{array}{c}
p_{\tau} \\
C-v p_{\varepsilon} \\
p_{\varepsilon}
\end{array}\right)
$$

In this case, the interface $x=0$ is characteristic $(\lambda=0$ is an eigenvalue of $\mathbf{A}(\mathbf{u}))$, and we have for each system one strictly positive and one strictly negative eigenvalue. We are going to study the coupling in two cases, transmitting either the conservative variables (condition (5) ) or the primitive variables $(\tau, v, p)$ (condition (77)). To justify the last choice, let $\mathbf{u}$ be a solution of system (17)-(18) containing two states separated by a contact discontinuity at $x=0, \mathbf{u}_{-} \equiv \mathbf{u}(0-, t)$ and $\mathbf{u}_{+} \equiv \mathbf{u}(0+, t)$. Then we have continuity of the 2-Riemann invariants $v, p$

$$
\left\{\begin{array}{l}
v_{-}=v_{+} \\
p\left(\tau_{-}, \varepsilon_{-}\right)=p\left(\tau_{+}, \varepsilon_{+}\right) .
\end{array}\right.
$$

When coupling the two systems (1) and (2) with $\mathbf{f}_{\alpha}$ given by (19), we may also want to transmit the velocity and the pressure. We will show that it corresponds to the coupling conditions (7) expressed in the primitive variables

$$
\mathbf{v}=(\tau, v, p)^{T} .
$$

Let us describe more precisely the change of variables

$$
\mathbf{u}=(\tau, v, e)^{T}=\varphi_{\alpha}(\mathbf{v}) .
$$

Since $e=\varepsilon+\frac{1}{2} v^{2}$, we assume all along this paper that the functions $p=p_{\alpha}(\tau, \varepsilon)$ may be inverted in $\varepsilon=\varepsilon_{\alpha}(\tau, p)$, which is the case for instance for a polytropic ideal gas satisfying a $\gamma$-law $p=(\gamma-1) \tau \varepsilon$, more generally, we assume $\frac{\partial p}{\partial \varepsilon}>0$.

\subsection{Coupled Riemann problem with transmission of primitive variables.}

This case is easier to deal with. Indeed, the Riemann problem for (17)-(18) is usually solved using primitive variables because the 'projection' of the wave curves on the $(v, p)$-plane are easily expressed. Moreover, this choice is consistent with what we have done in the isentropic case for the $p$-system. Finally this choice appears fairly natural from a physical point of view since Proposition 2 below shows that it corresponds to transmit $p$ and $v$, which are naturally transmitted when the two laws coincide.

Let $\mathbf{u}_{L}$ and $\mathbf{u}_{R}$ be two given states. We denote by $\mathscr{C}_{R}^{1}\left(\mathbf{u}_{L}\right)$ the 1 - wave curve consisting of states $\mathbf{u}$ which can be connected to $\mathbf{u}_{L}$ on the right by either a 1 -shock or a 1 -rarefaction wave corresponding to the equation of state $p=p_{R}(\tau, \varepsilon)$. Similarly, given a right state $\mathbf{u}_{R}$, we denote by $\check{\mathscr{C}}_{L}^{3}\left(\mathbf{u}_{R}\right)$ the (backward) 3 -wave curve consisting of left states $\mathbf{u}$ which can be connected to $\mathbf{u}_{R}$ by a 3 -shock or a 3-rarefaction wave corresponding to the equation of state $p=p_{L}(\tau, \varepsilon)$. We denote by $\mathbf{C}_{R}^{1}\left(\mathbf{v}_{L}\right)$ and $\check{\mathbf{C}}_{L}^{3}\left(\mathbf{v}_{R}\right)$ the 'projections' (in a sense to be made precise below) onto the $(v, p)$ plane of the wave curves $\mathscr{C}_{R}^{1}\left(\mathbf{u}_{L}\right)$ and $\check{\mathscr{C}}_{L}^{3}\left(\mathbf{u}_{R}\right)$ respectively. In fact $\mathbf{C}_{\alpha}^{i}\left(\mathbf{v}_{L}\right)$ is the 
projection of the $i$-wave curve curve $\varphi_{\alpha}^{-1}\left(\mathscr{C}^{i}\left(\mathbf{u}_{L}\right)\right)$ expressed in primitive variables $\mathbf{v}=(\tau, v, p)^{T}$ on the $(v, p)$-plane:

$$
\varphi_{\alpha}^{-1}\left(\mathscr{C}_{\alpha}^{i}\left(\mathbf{u}_{L}\right)\right)=\left\{\mathbf{v}=(\tau, v, p)^{T} ; \varphi_{\alpha}(\mathbf{v}) \in \mathscr{C}_{\alpha}^{i}\left(\mathbf{u}_{L}\right)\right\}
$$

and

$$
\mathbf{C}_{\alpha}^{i}\left(\mathbf{v}_{L}\right)=\left\{(v, p) ;(\tau, v, p)^{T} \in \varphi_{\alpha}^{-1}\left(\mathscr{C}_{\alpha}^{i}\left(\mathbf{u}_{L}\right)\right)\right\} .
$$

Similar definitions for the backward wave curve $\check{\mathbf{C}}_{\alpha}^{i}\left(\mathbf{v}_{R}\right)$ are in order.

We then make the following hypothesis:

$$
\left\{\begin{array}{l}
\text { for any pair of states }\left(\mathbf{u}_{\ell}, \mathbf{u}_{r}\right) \text {, the curves } \\
\mathbf{C}_{R}^{1}\left(\mathbf{v}_{\ell}\right) \text { and } \check{\mathbf{C}}_{L}^{3}\left(\mathbf{v}_{r}\right) \text { may intersect at one point at most. }
\end{array}\right.
$$

This assumption simply guarantees that the Riemann problem has a unique solution. It can also be expressed in terms of monotonicity of the corresponding curves, as illustrated in Figure 6. $\mathbf{C}_{\alpha}^{1}$ is decreasing and $\check{\mathbf{C}}_{\alpha}^{3}$ increasing, and we refer to [15] for precise assumptions on the equation of state which ensure this property (see however Remark 2 below).

Proposition 2. Assume the hypothesis (26). Then, in the case (24), the coupling conditions (7) lead to

$$
\left\{\begin{array}{l}
v(0-, t)=v(0+, t), \\
p(0-, t)=p(0+, t) .
\end{array}\right.
$$

In addition, the coupled Riemann problem has a unique solution (away from vacuum).

Proof. By using the structure of the solution of the whole Riemann problem for the gas dynamics equations with pressure law $p_{L}$, let us show that the condition $\mathbf{u}(0-, t) \in \mathcal{O}_{L}\left(\varphi_{L}(\mathbf{v}(0+, t))\right)$ means that

$$
(v, p)(0-, t) \in \check{\mathbf{C}}_{L}^{3}(\mathbf{v}(0+, t)) .
$$

Let us first make precise the states that are involved in the above expressions:

$$
\begin{gathered}
\mathbf{v}(0+, t)=(\tau(0+, t), v(0+, t), p(0+, t))^{T} \\
\varphi_{L}(\mathbf{v}(0+, t))=(\tau(0+, t), v(0+, t), e(0+, t))^{T}
\end{gathered}
$$

where $e(0+, t)=\varepsilon_{L}(\tau(0+, t), p(0+, t))+v(0+, t)^{2} / 2$,

$$
\mathbf{u}(0-, t)=(\tau(0-, t), v(0-, t), e(0-, t))^{T}
$$

and $p(0-, t)=p_{L}\left(\tau(0-, t), e(0-, t)-v(0-, t)^{2} / 2\right)$.

Then, by definition of the admissible set $\mathcal{O}_{L}$, there exists a state $\mathbf{u}_{-} \in \Omega$ such that

$$
\mathbf{u}(0-, t)=\mathbf{W}_{L}\left(0-; \mathbf{u}_{-}, \varphi_{L}(\mathbf{v}(0+, t))\right) .
$$

The $L$-Riemann problem between $\mathbf{u}_{-}$and $\varphi_{L}(\mathbf{v}(0+, t))$ is thus built with a $1 L$ -wave between $\mathbf{u}_{-}$and $\mathbf{u}(0-, t)$, a 2 -contact discontinuity at $x=0$ between $\mathbf{u}(0-, t)$ and a state $\mathbf{u}_{+}^{L}$, and a $3 L$-wave between $\mathbf{u}_{+}^{L}$ and $\varphi_{L}(\mathbf{v}(0+, t))$. This yields that $\left(v_{+}^{L}, p_{+}^{L}\right)=(v(0-, t), p(0-, t))$ and thus, after projection on the $(v, p)-$ plane, $(v, p)(0-, t))$ belongs to $\check{\mathbf{C}}_{L}^{3}(\mathbf{v}(0+))$. Similarly, the condition $\mathbf{u}(0+, t) \in \mathcal{O}_{R}\left(\varphi_{R}\right.$ $(\mathbf{v}(0-, t)))$ means that

$$
(v, p)(0+, t) \in \mathbf{C}_{R}^{1}(\mathbf{v}(0-)) .
$$



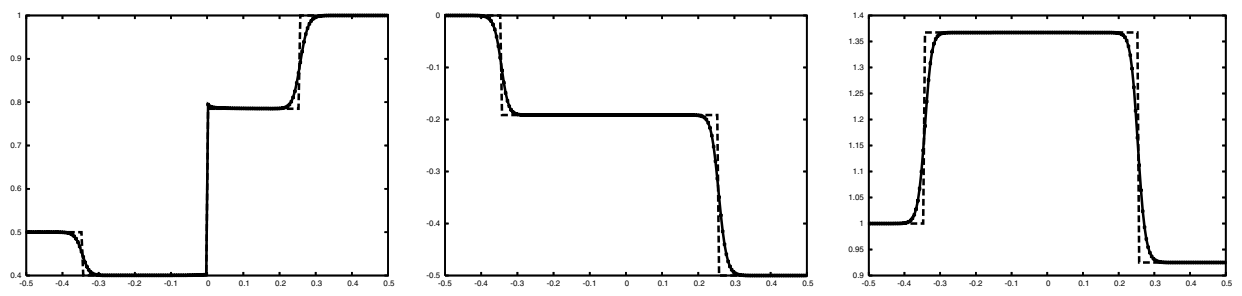

FiguRe 4. $\tau, v, p$ for the coupled Riemann problem for the Euler system with transmission of $\mathbf{v}=(\tau, v, p)$ : discontinuity of $\tau$, continuity of $v, p$ at $x=0$

If (26) holds, and away from vacuum, the curves $\mathbf{C}_{R}^{1}(\mathbf{v}(0-))$ and $\check{\mathbf{C}}_{L}^{3}(\mathbf{v}(0+))$ intersect at only one point in the $(v, p)$-plane (see Figure 6 left); then (28)-(29) imply that $(v, p)(0+, t)$ and $(v, p)(0-, t)$ must necessarily coincide since, in the $(v, p)$-plane, both $(v, p)(0+, t)$ and $(v, p)(0-, t)$ lie on both curves, which proves the lemma.

Finally, we can solve the coupled Riemann problem following the usual procedure, by first solving the systems of equations obtained by intersection of the projected curves on the $(v, p)$-plane (see [11]). The solution exists (if no vacuum appears) and is unique.

The result is illustrated in Figure 4 on a coupled Riemann problem for two $\gamma$-laws, $\gamma_{L}=1.4, \gamma_{R}=1.6$.

Remark 2. The curve $\mathscr{C}_{R}^{1}\left(\mathbf{u}_{\ell}\right)$ is tangent at $\mathbf{u}_{\ell}$ to the first eigenvector (see formula (21) $\mathbf{r}_{R, 1}\left(\mathbf{u}_{\ell}\right)=\left(-1,-C_{R}, p_{R}-C_{R} v\right)^{T}\left(\mathbf{u}_{\ell}\right)$. In primitive variables, the curve $\varphi_{R}^{-1}\left(\mathscr{C}_{R}^{1}\left(\mathbf{u}_{\ell}\right)\right)$ is tangent to $\varphi_{R}^{\prime}\left(\mathbf{v}_{\ell}\right)^{-1}\left(\mathbf{r}_{R, 1}\left(\mathbf{u}_{\ell}\right)\right)=\left(-1,-C_{R}, C_{R}^{2}\right)^{T}\left(\mathbf{u}_{\ell}\right)$ which is the 1-eigenvector in primitive variables and, by projection on the $(v, p)$-plane, $\mathbf{C}_{R}^{1}\left(\mathbf{v}_{\ell}\right)$ is tangent to the vector $\left(-C_{R}, C_{R}^{2}\right)^{T}\left(\mathbf{u}_{\ell}\right)$ or equivalently to $\left(-1, C_{R, \ell}\right)^{T}$ at state $\left(v_{\ell}, p_{\ell}\right)$. Similarly, $\check{\mathscr{C}}_{L}^{3}\left(\mathbf{v}_{r}\right)$ is tangent to $\left(1, C_{L, r}\right)^{T}$ at state $\left(v_{r}, p_{r}\right)$. The vectors $\left(-1, C_{R, \ell}\right)^{T}$ and $\left(1, C_{L, r}\right)^{T}$ are not colinear. Thus hypothesis (26) is satisfied at least for nearby states $\left(\mathbf{u}_{\ell}, \mathbf{u}_{r}\right)$. It may be globally satisfied for 'standard' equations of state.

3.3. Transmission of conservative variables. The polytropic ideal gas case. As already observed, the above derivation of the coupling condition in primitive variables was made easy by the usual way of solving the classical Riemann problem in the $(v, p)$-plane. If we now want to transmit the conservative variables, we must interpret the coupling conditions $\mathbf{u}(0-, t) \in \mathcal{O}_{L}(\mathbf{u}(0+, t))$ and $\mathbf{u}(0+, t) \in \mathcal{O}_{R}(\mathbf{u}(0-, t))$ in terms of conservative variables $(\tau, u, e)$. Again, the solution of the $L$-Riemann problem between a state $\mathbf{u}_{-}$and $\mathbf{u}(0+, t)$ is made of a $1 L$-wave between $\mathbf{u}_{-}$and $\mathbf{u}(0-, t)$, a $2 L$-contact discontinuity at $x=0$ between $\mathbf{u}(0-, t)$ and a state $\mathbf{u}_{+}^{(L)}$ and a $3 L$-wave between $\mathbf{u}_{+}^{(L)}$ and $\mathbf{u}(0+, t)$ (see Figure 5 , left).

We first make the simplifying assumption that the two pressure laws are $\gamma$-laws:

$$
p_{\alpha}=\left(\gamma_{\alpha}-1\right) \varepsilon / \tau, \gamma_{\alpha}>1 \text {. }
$$




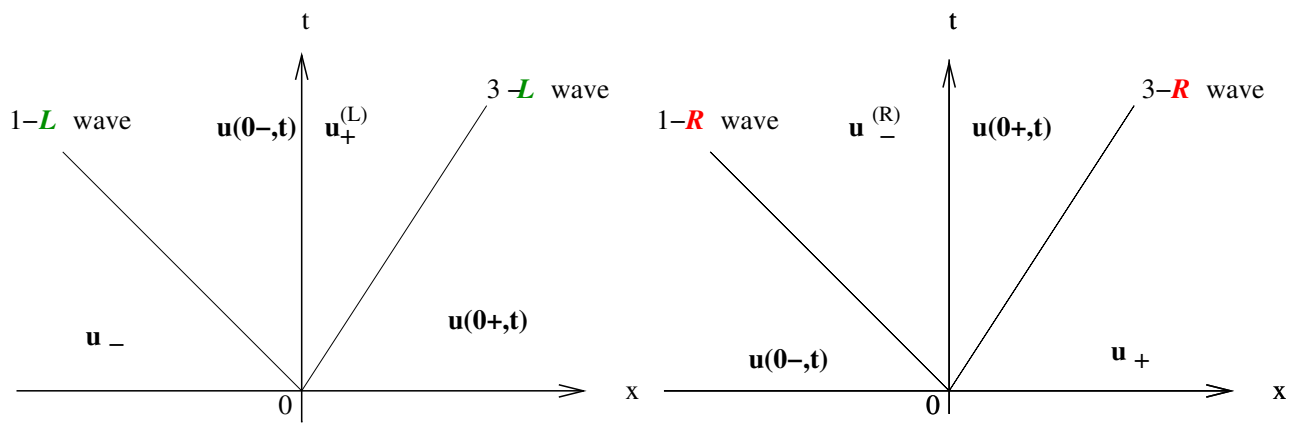

Figure 5. Coupling conditions (5) for the Euler system: $\mathbf{u}(0-, t) \in \mathcal{O}_{L}(\mathbf{u}(0+, t))($ left $), \mathbf{u}(0+, t) \in \mathcal{O}_{R}(\mathbf{u}(0-, t))$ (right)

We get that $\left(v_{+}^{(L)}, p_{+}^{(L)}\right)=(v(0-, t), p(0-, t))$, more precisely,

$$
v_{+}^{(L)}=v(0-, t)
$$

and

$$
p_{L}\left(\mathbf{u}_{+}^{(L)}\right)=p_{L}(\mathbf{u}(0-, t))
$$

and thus $\left(\gamma_{L}-1\right) \varepsilon_{+}^{(L)} / \tau_{+}^{(L)}=\left(\gamma_{L}-1\right) \varepsilon(0-, t) / \tau(0-, t)$, which implies

$$
\frac{\varepsilon_{+}^{(L)}}{\tau_{+}^{(L)}}=\frac{\varepsilon(0-, t)}{\tau(0-, t)} .
$$

Following the usual way of solving the Riemann problem, we 'project' the condition

$$
\mathbf{u}_{+}^{(L)} \in \check{\mathscr{C}}_{L}^{3}(\mathbf{u}(0+))
$$

on the $(v, p)$-plane.

Similarly, the solution of the $R$-Riemann problem between $\mathbf{u}(0-, t)$ and a state $\mathbf{u}_{+}$is made of a $1 R$-wave between $\mathbf{u}(0-, t)$ and a state $\mathbf{u}_{-}^{(R)}$, a $2 R$-contact discontinuity at $x=0$ between $\mathbf{u}_{-}^{(R)}$ and $\mathbf{u}(0+, t)$, and a $3 R$-wave between $\mathbf{u}(0+, t)$ and $\mathbf{u}+$ (see Figure 5, right). This yields that $\left(v_{-}^{(R)}, p_{-}^{(R)}\right)=(v(0+, t), p(0+, t))$, more precisely,

$$
v_{-}^{(R)}=v(0+, t)
$$

and

$$
p_{R}\left(\mathbf{u}_{-}^{(R)}\right)=p_{R}(\mathbf{u}(0+, t)),
$$

and thus

$$
\frac{\varepsilon_{-}^{(R)}}{\tau_{-}^{(R)}}=\frac{\varepsilon(0+, t)}{\tau(0+, t)} .
$$

Again we 'project' the condition

$$
\mathbf{u}_{-}^{(R)} \in \mathscr{C}_{R}^{1}(\mathbf{u}(0-))
$$

on the $(v, p)$-plane. Thus we have to meet the conditions

$$
M^{L}(0-)=\left(v(0-, t), p_{L}(\mathbf{u}(0-, t))\right) \in \check{\mathbf{C}}_{L}^{3}(\mathbf{v}(0+))
$$



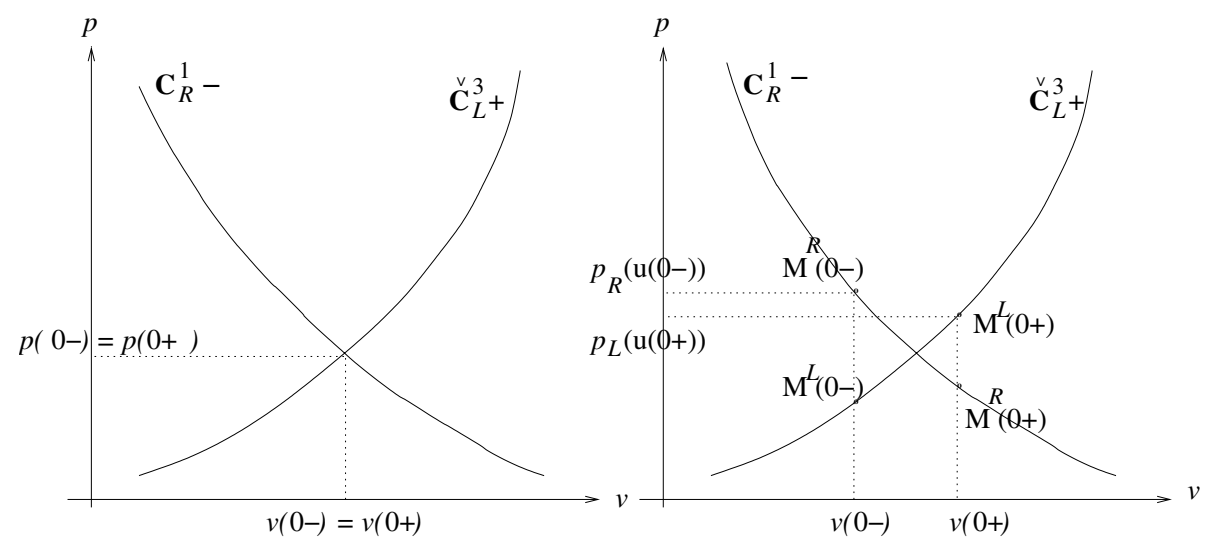

Figure 6. Projection of the wave curves: transmission of $\mathbf{v}$ (left)transmission of $\mathbf{u}$ (right), with $\mathbf{C}_{R}^{1}-=\mathbf{C}_{R}^{1}(\mathbf{v}(0-)), \check{\mathbf{C}}_{L}^{3}+=$ $\check{\mathbf{C}}_{L}^{3}(\mathbf{v}(0+))$

and

$$
M^{R}(0+)=\left(v(0+, t), p_{R}(\mathbf{u}(0+, t))\right) \in \mathbf{C}_{R}^{1}(\mathbf{v}(0-)),
$$

and we can no longer intersect the two curves in order to solve the problem. Figure 6 left (resp. right) illustrates the position of the projected wave curves when the coupling conditions (7) (resp. (5)) are satisfied. Indeed, since in the $(v, p)$-plane the curve

$$
\mathbf{C}_{R}^{1}(\mathbf{v}(0-))=\left\{(v, p) ;(\tau, v, p)^{T} \in \varphi_{R}^{-1}\left(\mathscr{C}_{R}^{1}(\mathbf{u}(0-))\right)\right\},
$$

starts from point $M^{R}(0-)=\left(v(0-, t), p_{R}(\mathbf{u}(0-, t))\right)$, and not, as it did in the previous case, from $M^{L}(0-)=\left(v(0-, t), p_{L}(\mathbf{u}(0-, t))\right)$ (see Figure 6 right), then $\check{\mathbf{C}}_{L}^{3}(\mathbf{v}(0+))$ starts from $M^{L}(0+)=\left(v(0+, t), p_{L}(\mathbf{u}(0+, t))\right)$. The intersection of the two curves no longer solves the problem.

In fact, the $(v, p)$-plane is not well suited, since $p$ is no longer a transmitted variable. For two $\gamma$-laws, because of (33)-(37), we can think of the plane $(v, \pi=$ $\varepsilon / \tau)$, since $\pi$ is a variable independent of the pressure law. Following the above arguments and projecting on the $(v, \pi)$-plane will lead to the intersection of two curves. More precisely,

Proposition 3. Assuming (30), the coupling conditions (5) lead to

$$
\left\{\begin{array}{l}
v(0-, t)=v(0+, t), \\
\frac{\varepsilon}{\tau}(0-, t)=\frac{\varepsilon}{\tau}(0+, t) .
\end{array}\right.
$$

Proof. Define

$$
\mathbf{w}=(\tau, v, \pi)^{T}
$$

where

$$
\pi=\frac{\varepsilon}{\tau}
$$

Then define the mapping $\tilde{\varphi}$ by $\mathbf{u}=\tilde{\varphi}(\mathbf{w})$, and let

$$
\tilde{\mathbf{C}}_{R}^{1}(\mathbf{w}(0-))=\left\{(v, \pi) ;(\tau, v, \pi)^{T} \in \tilde{\varphi}_{R}^{-1}\left(\mathscr{C}_{R}^{1}(\mathbf{u}(0-))\right)\right\},
$$



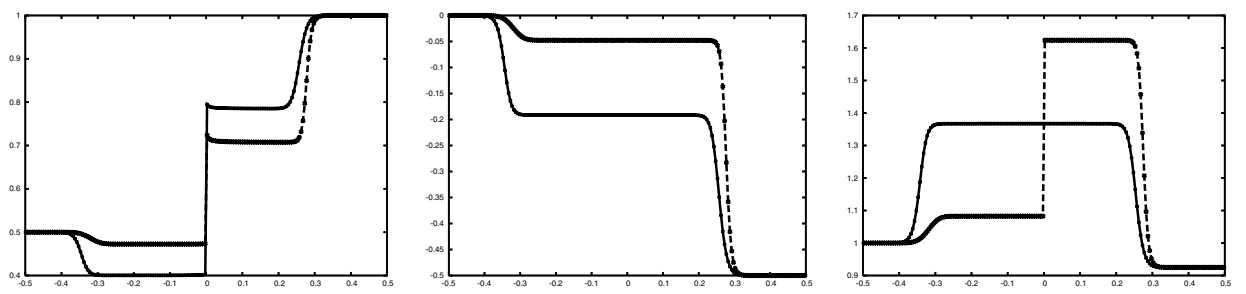

Figure 7. $\tau, v, p$ in the transmission of $\mathbf{u}=(\tau, v, e)$ vs. $\mathbf{v}=$ $(\tau, v, p)$ the for Euler system: Discontinuity of $\tau$ - Continuity of $v$ - Continuity of $p$ for $\mathbf{v} /$ Discontinuity of $p$ for $\mathbf{u}$

with a similar definition for the backward curve $\check{\tilde{\mathbf{C}}}_{L}^{3}(\mathbf{w}(0+))$. The projection on the $(v, \pi)$-plane of (34), (38) together with (31), (37) (33), (37) then yields

$$
\begin{aligned}
& (v(0-, t), \pi(0-, t)) \in \check{\tilde{\mathbf{C}}}_{L}^{3}(\mathbf{w}(0+)), \\
& (v(0+, t), \pi(0+, t)) \in \tilde{\mathbf{C}}_{R}^{1}(\mathbf{w}(0-)) .
\end{aligned}
$$

Now, assuming (26) implies that $\tilde{\mathbf{C}}_{R}^{1}(\mathbf{w}(0-))$ and $\tilde{\mathbf{C}}_{L}^{3}(\mathbf{w}(0+))$ intersect at only one point, since the change of variables $\pi$ preserves the respective monotonicity of the curves. We get

$$
\left\{\begin{array}{l}
v(0-, t)=v(0+, t), \\
\pi(0-, t)=\pi(0+, t),
\end{array}\right.
$$

which is the desired result.

The coupled Riemann problem is then solved in the same way as before since we can parametrize each wave curve by $\pi$ instead of $p$.

A coupled Riemann problem is illustrated in Figure 7 where the transmissions of conservative and of physical variables are compared.

We can easily extend the result to the case of pressure laws which can be written as a function of one dependent variable $\pi=\pi(\tau, \varepsilon)$, i.e. such that

$$
p_{\alpha}(\tau, \varepsilon)=\bar{p}_{\alpha}(\pi(\tau, \varepsilon)) \text {. }
$$

The above argument will show that $(v, \pi)$ is continuous at the interface $x=0$.

Now consider two thermally perfect gases, such that $p \tau=R T(\varepsilon)$ ( $T$ is the temperature). The pressure law is of the following form:

$$
p=p(\tau, \varepsilon)=\tilde{p}(\tau, \pi)
$$

again with $\pi=\varepsilon / \tau$ and satisfying the identity

$$
p=-\tau \tilde{p}_{\tau}+\pi \tilde{p}_{\pi} .
$$

We thus have

$$
-\frac{p_{\tau}}{p_{\varepsilon}}=\frac{p}{\tilde{p}_{\pi}}
$$

or we can also write

$$
-\frac{p_{\tau}}{p_{\varepsilon}}=\frac{T(\varepsilon)}{\tau T^{\prime}(\varepsilon)} .
$$

If this quantity is independent of the closure law, we can take it as a dependent variable and conclude that it will be continuous at the interface together with the velocity. 
Remark 3. Assuming a $\gamma$-law yields that the eigenvector $\mathbf{r}_{2}(\mathbf{u})$ in (21) can be chosen as $(1,0, \varepsilon / \tau)^{T}=(1,0, \pi)^{T}$. Thus the function $\mathbf{r}_{2}(\mathbf{u})$ does not depend on the pressure law and (39) means that $\mathbf{r}_{L, 2}(\mathbf{u}(0-, t))=\mathbf{r}_{R, 2}(\mathbf{u}(0+, t))$. In the linearized approach, linearizing the left problem at $\mathbf{u}(0-, t)$ and the right problem at $\mathbf{u}(0+, t)$, and coupling these two problems, the necessary condition (3.19) of 13 requiring $\operatorname{dim} E=1$ is indeed satisfied since $E=\left[\mathbf{r}_{R, 1} \mathbf{r}_{R, 2}\right] \cap\left[\mathbf{r}_{L, 2} \mathbf{r}_{L, 3}\right]=\mathbb{R} \mathbf{r}_{L, 2}=\mathbb{R} \mathbf{r}_{R, 2}$, where the notation $\left[\mathbf{r}_{\alpha, i} \mathbf{r}_{\alpha, j}\right]$ denotes the vector space spanned by the vectors $\mathbf{r}_{\alpha, i}, \mathbf{r}_{\alpha, j}$.

The above section brings some precisions to the corresponding section (Section 4) of [13], where it was not specified that the coupling was achieved in primitive variables.

3.4. Transmission of conservative variables. The general case. Let us first see that the velocity need not be continuous for general pressure laws. Assume first that $v$ is continuous at the interface

$$
v(0+, t)=v(0-, t) .
$$

If, for instance, the $3 L$-wave in the $L$-Riemann problem and the $1 R$-wave in the $R$-Riemann problem are both shocks (see Figure 5), we get from the RankineHugoniot relations concerning the $3 L$-shock

$$
p_{L}(\mathbf{u}(0-, t))=p_{L}(\mathbf{u}(0+, t))
$$

and similarly

$$
p_{R}(\mathbf{u}(0-, t))=p_{R}(\mathbf{u}(0+, t)) .
$$

For instance to get the first formula, we write the Rankine-Hugoniot relation concerning the $3 L-$ shock

$$
-\sigma_{3, L}\left(v(0+, t)-v_{+}^{(L)}\right)+p_{L}(\mathbf{u}(0+, t))-p_{L}\left(\mathbf{u}_{+}^{L}\right)=0,
$$

with the invariance of $v, p$ at the contact discontinuity

$$
v_{+}^{(L)}=v(0-, t), p_{L}\left(\mathbf{u}_{+}^{L}\right)=p_{L}(\mathbf{u}(0-, t))
$$

(similarly the $1 R$-shock relation for the second formula).

Consider for instance two pressure laws of Grüneisen type

$$
p_{\alpha}(\tau, \varepsilon)=\left(\gamma_{\alpha}-1\right) \frac{\varepsilon}{\tau}+d_{\alpha}^{2}\left(\frac{1}{\tau}-\frac{1}{\tau_{r e f, \alpha}}\right), \alpha=L, R .
$$

For (43), the continuity of $p_{R}$ and $p_{L}$ yields

$$
\left(\gamma_{\alpha}-1\right)\left(\frac{\varepsilon}{\tau}(0+, t)-\frac{\varepsilon}{\tau}(0-, t)\right)+d_{\alpha}^{2}\left(\frac{1}{\tau(0+, t)}-\frac{1}{\tau(0-, t)}\right)=0, \alpha=L, R,
$$

which implies, as soon as

$$
\frac{d_{L}^{2}}{\gamma_{L}-1} \neq \frac{d_{R}^{2}}{\gamma_{R}-1},
$$

that $\frac{\varepsilon}{\tau}(0-, t)=\frac{\varepsilon}{\tau}(0+, t)$ and $\tau(0-, t)=\tau(0+, t)$ so that

$$
\varepsilon(0-, t)=\varepsilon(0+, t), \tau(0-, t)=\tau(0+, t) .
$$



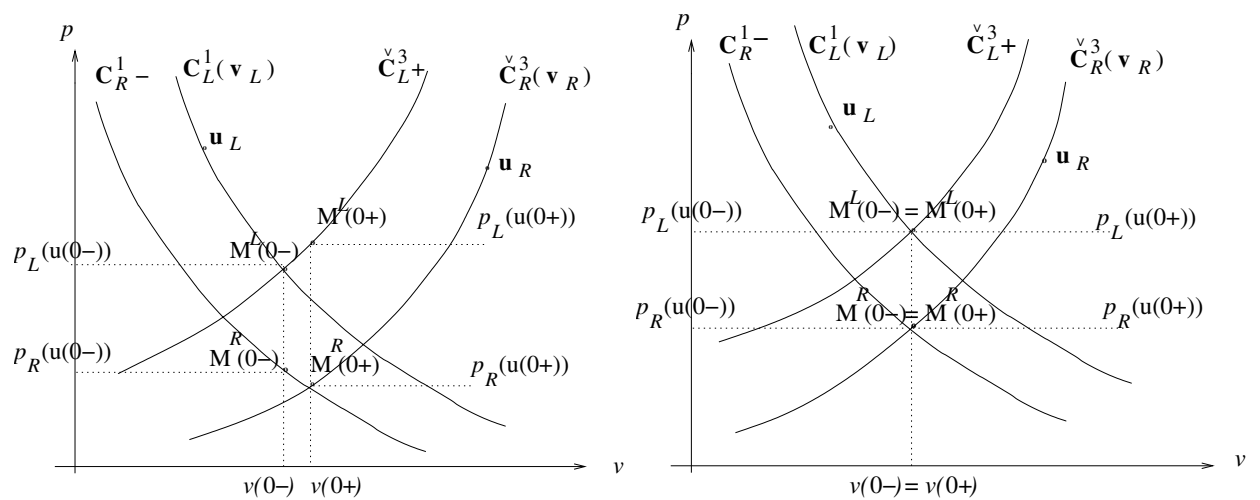

FiguRe 8. Coupled Riemann problem, projection of the wave curves: general case (left), special case (right), with $\mathbf{C}_{R}^{1}-=$ $\mathbf{C}_{R}^{1}(\mathbf{v}(0-)), \check{\mathbf{C}}_{L}^{3}+=\check{\mathbf{C}}_{L}^{3}(\mathbf{v}(0+))$

Remark 4. Thus, in the case (44), the velocity need not be continuous, and if the velocity is continuous, the whole state is continuous at the interface $\mathbf{u}(0-, t)=$ $\mathbf{u}(0+, t)$ (but $p_{L}(\mathbf{u}(0-, t)) \neq p_{R}(\mathbf{u}(0+, t))$ ). That may happen with particular given states $\mathbf{u}_{L}, \mathbf{u}_{R}$. Indeed if the coupled Riemann problem is solved with $\mathbf{u}(0-, t)=$ $\mathbf{u}(0+, t)$ noted $\mathbf{u}(0)=\left(\tau_{0}, v_{0}, e_{0}\right)$, then since $\mathbf{u}(0)=\mathbf{u}(0-) \in \mathscr{C}_{L}^{1}\left(\mathbf{u}_{L}\right)$ and $\mathbf{u}(0)=$ $\mathbf{u}(0+) \in \check{\mathscr{C}}_{R}^{3}\left(\mathbf{u}_{R}\right)$, the two curves in $\mathbb{R}^{3}$ necessarily intersect, not only their projection on a plane. For instance, the projection of these curves on the plane $(v, \varepsilon / \tau)$ determines $\left(v_{0}, \varepsilon_{0} / \tau_{0}\right)$, and their projection on the plane $(\tau, v)$ determines $\left(\tau_{0}, v_{0}\right)$. Assume $\mathbf{u}(0)$ is completely determined. Then the Hugoniot curves $\mathcal{H}_{\alpha}(\tau, p)=0$ are hyperbolas (cf. [11, Chapter II, Section 2, Example 2.2) and may be parametrized by $p$ : $\tau=h_{\alpha}\left(p ; \tau_{a}, p_{a}\right)$ for a Hugoniot curve with center $a$. This provides two relations $\tau_{0}=h_{L}\left(p_{L}\left(\mathbf{u}_{0}\right) ; \tau_{L}, p_{L}\right)=h_{R}\left(p_{R}\left(\mathbf{u}_{0}\right) ; \tau_{R}, p_{R}\right)$ where $p_{L}=p_{L}\left(\mathbf{u}_{L}\right), p_{R}=$ $p_{R}\left(\mathbf{u}_{R}\right)$. Hence $\mathbf{u}_{L}, \mathbf{u}_{R}$ should be such that $\left(\tau_{L}, p_{L}\right)$ and $\left(\tau_{R}, p_{R}\right)$ satisfy the identity $h_{L}\left(p_{L}\left(\mathbf{u}_{0}\right) ; \tau_{L}, p_{L}\right)=h_{R}\left(p_{R}\left(\mathbf{u}_{0}\right) ; \tau_{R}, p_{R}\right)$.

We consider in Figure 8 states and wave curves corresponding to a coupled Riemann problem for which the $3 L-$ and the $1 R$-waves are both shocks (cf. Figure 5). Let $M^{L}(0-)$ denote the point $\left(v(0-, t), p_{L}(\mathbf{u}(0-, t))\right)$ and $M^{R}(0+)$ the point $\left(v(0+, t), p_{R}(\mathbf{u}(0+, t))\right)$. The point $M^{L}(0-)$ (resp. $\left.M^{R}(0+)\right)$ is the projection of $\mathbf{u}(0-, t)$ (resp. $\mathbf{u}(0+, t))$ on the $(v, p)$-plane. In the above mentioned particular case, the curves $\mathbf{C}_{L}^{1}\left(\mathbf{u}_{L}\right)$ and $\check{\mathbf{C}}_{R}^{3}\left(\mathbf{u}_{R}\right)$ intersect at a point for which the states $\mathbf{u}(0-, t), \mathbf{u}(0+, t)$ do coincide. The two cases are illustrated in Figure 8

Now if

$$
\frac{d_{L}^{2}}{\gamma_{L}-1}=\frac{d_{R}^{2}}{\gamma_{R}-1} \equiv \frac{d^{2}}{\gamma-1},
$$

we notice that the above computations only give one relation linking $(\tau, \varepsilon / \tau)(0+, t)$ and $(\tau, \varepsilon / \tau)(0-, t)$. However, we can prove

Proposition 4. Assuming (43) with (45), the coupling conditions (5) lead to

$$
\left\{\begin{array}{l}
v(0-, t)=v(0+, t), \\
\left(\frac{\varepsilon}{\tau}+\left(\frac{d^{2}}{\gamma-1}\right) \frac{1}{\tau}\right)(0-, t)=\left(\frac{\varepsilon}{\tau}+\left(\frac{d^{2}}{\gamma-1}\right) \frac{1}{\tau}\right)(0+, t) .
\end{array}\right.
$$


Proof. Note that for two $\gamma$-laws $\left(d_{\alpha}^{2}=0\right)$, we have indeed seen that the coupling yields the continuity of $v$ and $\pi=\frac{\varepsilon}{\tau}$ by projecting on the $(v, \pi)$-plane. Assuming (43) and (45), we note that the quantity

$$
\omega_{\alpha} \equiv \frac{1}{\gamma_{\alpha}-1}\left(p+\frac{d_{\alpha}^{2}}{\tau_{r e f, \alpha}}\right)
$$

now plays a particular role since it satisfes

$$
\omega_{\alpha}=\frac{\varepsilon}{\tau}+\left(\frac{d_{\alpha}^{2}}{\gamma_{\alpha}-1}\right) \frac{1}{\tau}
$$

and thus does not depend on $\alpha$ if (45) holds; let us note it $\omega$. We can project on the $(v, \omega)$-plane, parametrize the projected wave curves by $\omega$ since $p \rightarrow \omega$ is an isomorphism, and following the same arguments as in the proof of Proposition 3, obtain the continuity of $v$ and $\omega$ at the interface.

Again, assuming (43) yields that the eigenvector $\mathbf{r}_{2}(\mathbf{u})$ in (21) is parallel to $(1,0,-\omega)^{T}$ and does not depend on the pressure law. Then (46) implies that $\mathbf{r}_{L, 2}(\mathbf{u}(0-, t))=\mathbf{r}_{R, 2}(\mathbf{u}(0+, t))$ (cf. Remark 3).

Remark 5. We can try to make explicit the quantities which are transmitted at the interface for more general pressure laws, since, in a heuristic way, we can say that 'two quantities are transmitted'. In fact these quantities are not explicit physical quantities in general, in particular the velocity is not necessarily continuous. For more general pressure laws, assuming for instance that $(\tau, v, \varepsilon)(0-, t)$ is known, and for some given $t$, the usual 'projection' on the $(v, p)$-plane, assuming that the 'projected' curves can be parametrized by $p$, provides a system of two equations in three unknowns $(\tau, v, \varepsilon)(0+, t)$, which can be interpreted as the intersection of two surfaces in $\mathbb{R}^{3}$; heuristically, this gives a curve. In the case we have already considered of two $\gamma$-laws (30), in variables $\mathbf{w}=(\tau, v, \pi)$, easy computations show that this curve is a straight line (39) intersection of two planes, parallel to the $\tau$-axis. In the case (43)-(44), we can say that the intersection of the curve with plane $v(0+, t)=v(0-, t)$ is a point, so that the curve is not contained in this plane. To make explicit the transmitted quantity means to find a change of variables, say $\tilde{\mathbf{w}} \in \mathbb{R}^{3}$, such that in these variables, the curve is a line parallel to one axis, say $\tilde{w}_{3}$, so that the quantities $\left(\tilde{w}_{1}, \tilde{w}_{2}\right)$ are continuous. We have been able to find it for (43)-(45). The coupled Riemann problem can then be solved, however the physical meaning of the transmission is not clear.

\section{Coupling Lagrangian systems of Different Dimensions}

4.1. The $p$-system and the Euler system. We consider the $p$-system (11) in the left half-plane and the Euler system in Lagrangian coordinates (18) in the right half-plane (using in this section capital letters to distinguish the variables of the larger system)

$$
\begin{gathered}
\frac{\partial \mathbf{u}}{\partial t}+\frac{\partial}{\partial x} \mathbf{f}_{L}(\mathbf{u})=\mathbf{0}, x<0, \mathbf{u}=(\tau, v)^{T}, \mathbf{f}_{L}(\mathbf{u})=(-v, p)^{T}, p=p_{L}(\tau), \\
\frac{\partial \mathbf{U}}{\partial t}+\frac{\partial}{\partial x} \mathbf{F}_{R}(\mathbf{U})=\mathbf{0}, x>0, \mathbf{U}=(\tau, v, e)^{T}, \mathbf{F}_{R}(\mathbf{U})=(-v, p, p v)^{T}, p=p_{R}(\tau, \varepsilon) .
\end{gathered}
$$

The dimensions of the two systems are now different, but the physical context helps to give a meaning to the coupling since some state variables such as the specific 

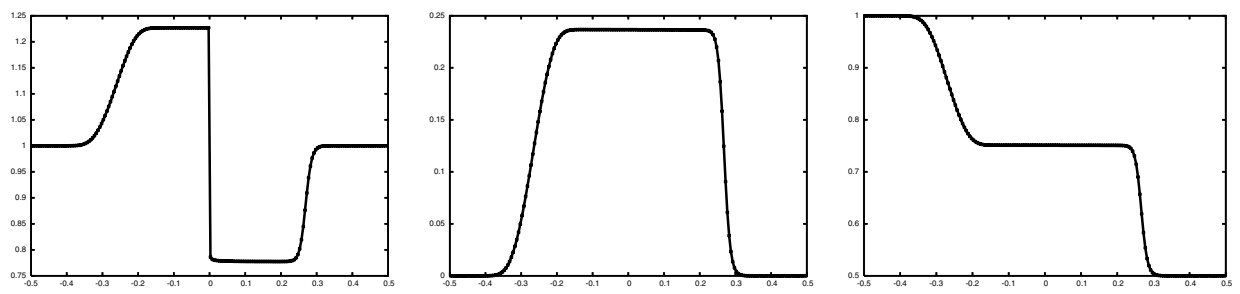

Figure 9. $\tau, v, p$ for the transmission of $\mathbf{v}=(v, p), p$-system in $x<0$ and Euler then system in $x>0$

volume $\tau$, velocity $v$ or pressure $p$ are defined for each model. Hence, we write coupling conditions using the variables $(v, p)$ that are common to the two systems and which we have seen are good candidates for both. The idea is to reconstruct the missing variable for the smaller system in such a way that we may transmit (i.e. have continuity of) the velocity and the pressure.

Indeed, we can lift $\mathbf{v}=(v, p)^{T}$ by reconstructing $\tau$ when we transmit from the left to the right

$$
\mathbf{v}=(v, p)^{T} \rightarrow \mathcal{L}(\mathbf{v})=(\tau, v, p)^{T}, \tau=\tau_{L}(p),
$$

where $p \rightarrow \tau_{L}(p)$ is the inverse of $p_{L}(\tau)$. We easily project $\mathbf{V}$ when we transmit from the right to the left

$$
\mathbf{V}=(\tau, v, p)^{T} \rightarrow \mathcal{P}(\mathbf{V})=(v, p)^{T} .
$$

The coupling conditions naturally write

$$
\left\{\begin{array}{l}
\mathbf{u}(0-, t) \in \mathcal{O}_{L}\left(\varphi_{L}(\mathcal{P}(\mathbf{V}(0+, t)))\right) \\
\mathbf{U}(0+, t) \in \mathcal{O}_{R}\left(\varphi_{R}(\mathcal{L}(\mathbf{v}(0-, t)))\right),
\end{array}\right.
$$

Here $\varphi_{L}(\mathbf{v})=\mathbf{u}$ and $\varphi_{R}(\mathbf{V})=\mathbf{U}$ are the previously defined admissible changes of variables (15) and (25).

Proposition 5. Defining $\mathcal{L}, \mathcal{P}$ by (47) and (48), the coupling conditions (49) lead to

$$
\left\{\begin{array}{l}
v(0-, t)=v(0+, t), \\
p(0-, t)=p(0+, t),
\end{array}\right.
$$

and the solution of the coupled Riemann problem is unique.

Proof. We express the Riemann problems associated to (49) using the variables $v$ and $p$. First $\mathbf{v}(0-, t)$ is connected to $\mathcal{P}(\mathbf{V}(0+, t))$ by a $2 L$-wave for the $p$-system. Then $\mathcal{L}(\mathbf{v}(0-, t))$ is connected to $\mathbf{V}(0+, t)$ by a $1 R$-wave for the Euler system, we project the corresponding $1 R$-wave curve on the $(v, p)$-plane, and its intersection with the $2 L$-wave curve for the $p$-system has only one intersection point, so that $\mathbf{v}(0-, t)=\mathcal{P}(\mathbf{V}(0+, t))$ and the result follows. We have implicitly assumed in the proof that the analogue of (26) holds.

The result is illustrated in Figure 9 with a $\gamma$-law $p_{L}(\tau)=\tau^{\gamma}, p_{R}(\tau, \varepsilon)=(\gamma-$ 1) $\varepsilon / \tau$, with $\gamma=1$.4. We note that $\tau$ is discontinuous whereas the continuity of $v, p$ at $x=0$ is indeed ensured.

We may also interpret the above coupling procedure by increasing the dimension of the smaller system in order to couple systems of the same dimension. 
4.2. Interpretation. In fact the previous approach of lifting and projection can be interpreted by adding an equation to the small system with two conservation laws

$$
\left\{\begin{array}{l}
\frac{\partial \tau}{\partial t}-\frac{\partial v}{\partial x}=0 \\
\frac{\partial v}{\partial t}+\frac{\partial p}{\partial x}=0 \\
\frac{\partial p}{\partial t}-p^{\prime}(\tau) \frac{\partial v}{\partial x}=0
\end{array}\right.
$$

which we write

$$
\frac{\partial \mathbf{V}}{\partial t}+B(\mathbf{V}) \frac{\partial \mathbf{V}}{\partial x}=0
$$

where $\mathbf{V}=(\tau, v, p)^{T}$. Only the third equation is in nonconservative form, but it is clearly redundant since $p=p(\tau)$, so the matrix $B(\mathbf{V})$ of the system

$$
B(\mathbf{V})=\left(\begin{array}{ccc}
0 & -1 & 0 \\
0 & 0 & 1 \\
0 & -p^{\prime}(\tau) & 0
\end{array}\right)
$$

is noninvertible. We have added to the existing eigenvalues $\lambda_{1}=-c, \lambda_{3}=c$, $c=\sqrt{-p^{\prime}(\tau)}$, a new eigenvalue which is precisely $\lambda_{2}=0$. The eigenvectors (in variables $\mathbf{V})$ are $\mathbf{s}_{2}=(1,0,0)^{T}, \mathbf{s}_{1}=\left(1, c,-c^{2}\right)^{T}$, and $\mathbf{s}_{3}=\left(1,-c,-c^{2}\right)^{T}$. These eigenvectors are presently the eigenvectors of the matrix of the full Euler system written in primitive variables $\mathbf{V}=(\tau, v, p)^{T}$, but with $C^{2}=p p_{\varepsilon}-p_{\tau}$. In fact, the corresponding third equation for the full Euler system in primitive variables is

$$
\frac{\partial p}{\partial t}+\left(p p_{\varepsilon}-p_{\tau}\right) \frac{\partial v}{\partial x}=0 .
$$

The Riemann invariants associated to 0 are $v, p$.

The only nonconservative product in the third equation of (51) can be defined through the first conservative equations. Indeed, $v$ and $p$ are continuous across a 2-discontinuity, and if $v$ and $p$ are discontinuous across a $1-$ or 3 -wave, the product $p^{\prime}(\tau) \frac{\partial v}{\partial x}$ is naturally defined by

$$
-p^{\prime}(\tau) \frac{\partial v}{\partial x}=\sigma^{2} \frac{\partial v}{\partial x}
$$

if $\sigma$ is the speed of propagation of the discontinuity, since we have $\sigma[p]=\sigma^{2}[v]$, where [.] denotes as usual the jump. So the augmented $p$-system (51) is a barotropic Euler system in which $p_{\varepsilon}=0$.

We can uniquely define a solution of the Riemann problem for (51) and initial data $\mathbf{V}_{L}=\left(\tau_{L}, v_{L}, p_{L}\right)^{T}, \mathbf{V}_{R}=\left(\tau_{R}, v_{R}, p_{R}\right)^{T}$. The initial data are supposed to satisfy $p=p(\tau)$, i.e. $p_{L}=p\left(\tau_{L}\right), p_{R}=p\left(\tau_{R}\right)$, but it is not necessary in what follows. A priori, the solution consists of a 1-wave between $\mathbf{V}_{L}$ and some state $\mathbf{V}_{L}^{*}$, a 2discontinuity between $\mathbf{V}_{L}^{*}$ and some state $\mathbf{V}_{R}^{*}$, and a 3 -wave between $\mathbf{V}_{R}^{*}$ and $\mathbf{V}_{R}$. Since $v, p$ are continuous across the 2 -discontinuity, i.e. $x=0$, the common value $\left(v^{*}, p^{*}\right)$ is determined as usual by the intersection of the projection of the $1-$ and 3 -wave curves on the $(v, p)$-plane, and it is the intermediate state in the solution of the Riemann problem for the $p$-system (solved in variable $(v, p)$ by parametrizing the wave curves by $p$ as we have seen in section 2.3, for the data $\left.\left(v_{L}, p_{L}\right),\left(v_{R}, p_{R}\right)\right)$. Now we have $p^{*}=p\left(\tau^{*}\right)$ so that $\mathbf{V}$ is continuous, $\mathbf{W}\left(0 \pm ; \mathbf{V}_{L}, \mathbf{V}_{R}\right)=\left(\tau^{*}, v^{*}, p^{*}\right)^{T}$. 
The coupling of (51) in $x<0$ with the Euler system (17) in $x>0$ is done through the condition

$$
\left\{\begin{aligned}
\mathbf{V}(0-, t) & \in \mathcal{O}_{L}(\mathbf{V}(0+, t)), \\
\mathbf{U}(0+, t) & \in \mathcal{O}_{R}\left(\varphi_{R}(\mathbf{V}(0-, t))\right) .
\end{aligned}\right.
$$

Proposition 6. The coupling conditions (52) are equivalent to (50).

Proof. Expressing the condition $\mathbf{V}(0-, t) \in \mathcal{O}_{L}(\mathbf{V}(0+, t))$ gives that $(v, p)(0-, t)$ belongs to the projection on the $(v, p)$-plane of the (backward) 3-wave curve, and the projection passes through $(v, p)(0+, t)$. Note that the given value $\tau(0+, t)$ does not satisfy $p=p(\tau)$, but as we have noticed above, it does not prevent us from solving the Riemann problem for system (51).

Similarly expressing the condition $\mathbf{U}(0+, t) \in \mathcal{O}_{R}\left(\varphi_{R}(\mathbf{V}(0-, t))\right)$ gives the fact that the point $(v, p)(0+, t)$ belongs to the projection of the 1 -wave curve through $(v, p)(0-, t)$. Again, the two curves intersect at only one point in the $(v, p)-$ plane.

If the initial states satisfy $p_{L}=p_{L}\left(\tau_{L}\right), p_{R}=p_{R}\left(\tau_{R}\right)$ the two solutions of the coupled Riemann problem satisfying (52) or (501) coincide.

4.3. Another interpretation: coupling of the isentropic and full systems. We now consider the $p$-system in the left half-plane as the isentropic model of gas dynamics, thus augmented by $\frac{\partial s}{\partial t}=0$, and we write

$$
\left\{\begin{array}{l}
\frac{\partial \tau}{\partial t}-\frac{\partial v}{\partial x}=0 \\
\frac{\partial v}{\partial t}+\frac{\partial p}{\partial x}=0 \\
\frac{\partial s}{\partial t}=0
\end{array}\right.
$$

assuming

$$
p=p\left(\tau, s_{0}\right)
$$

for some fixed value $s_{0}$ of the specific entropy where $p=p(\tau, s)$ is the equation of state of the fluid expressed in terms of $\tau$ and $s$. For instance for a perfect gas we have $p(\tau, s)=(\gamma-1) \exp \left(\left(s-s_{0}\right) / C_{v}\right) \tau^{-\gamma}$.

We then consider the coupled problem

$$
\left\{\begin{array}{c}
\frac{\partial \mathbf{W}}{\partial t}+\frac{\partial}{\partial x} \mathbf{F}_{L}(\mathbf{W})=\mathbf{0}, x<0, \\
\frac{\partial \mathbf{U}}{\partial t}+\frac{\partial}{\partial x} \mathbf{F}_{R}(\mathbf{U})=\mathbf{0}, x>0
\end{array}\right.
$$

where

$$
\mathbf{W}=(\tau, v, s)^{T}, \mathbf{U}=(\tau, v, e)^{T},
$$

and

$$
\mathbf{F}_{L}(\mathbf{W})=(-v, p, 0)^{T}, \mathbf{F}_{R}(\mathbf{U})=(-v, p, p v)^{T},
$$

so that the systems now have the same size.

We set $\tilde{\mathbf{W}}=(v, p, s)^{T}$, and define an admissible change of variables $\psi$ by $\psi(\tilde{\mathbf{W}})=\mathbf{W}$. More precisely $\psi_{L}(\tilde{\mathbf{W}})=\mathbf{W}=\left(v, \tau_{L}(p), s\right)^{T}$ is an admissible change of variables for (53) with pressure law $p=p_{L}(\tau)$ and $\tau_{L}(p)$ its inverse. Now, for 
the Euler system, the mapping $\mathbf{V}=(\tau, v, p)^{T} \rightarrow \mathbf{U}=\varphi_{R}(\mathbf{V})=(\tau, v, e)^{T}$ is an admissible change of variables, and so is $\tilde{\mathbf{W}}=(v, p, s)^{T} \rightarrow \tilde{\varphi}_{R}(\tilde{\mathbf{W}}) \equiv \mathbf{V}=(\tau, v, p)^{T}$, when assuming that $s=s(\tau, p)$ satisfies $\partial_{\tau} s \neq 0$. For instance, in the case of a $\gamma$-law, we have seen that $p_{R}(\tau, s)=\left(\gamma_{R}-1\right) \tau^{-\gamma_{R}} \exp \left(\left(s-s_{0}\right) / C_{v}\right)$, thus $s_{R}(\tau, p)=$ $s_{0}+C_{v} \log \left(p \tau^{\gamma_{R}} /\left(\gamma_{R}-1\right)\right)$. Therefore we can write $\left.\mathbf{U}=\varphi_{R}\left(\tilde{\varphi}_{R}(\tilde{\mathbf{W}})\right) \equiv \tilde{\psi}_{R}(\tilde{\mathbf{W}})\right)$ and it is admissible.

For the coupling problem (54), we take the following coupling conditions:

$$
\left\{\begin{array}{l}
\mathbf{W}(0-, t) \in \mathcal{O}_{L}\left(\psi_{L}(\tilde{\mathbf{W}}(0+, t))\right) \\
\mathbf{U}(0+, t) \in \mathcal{O}_{R}\left(\tilde{\psi}_{R}(\tilde{\mathbf{W}}(0-, t))\right)
\end{array}\right.
$$

where $\tilde{\mathbf{W}}(0+)=\left(\tilde{\psi}_{R}\right)^{-1}(\mathbf{U}(0+))=(v, p, s)^{T}(0+)$, with $p(0+)=p_{R}(\tau(0+), \varepsilon(0+))$, and $\tilde{\mathbf{W}}(0-)=\left(\psi_{L}\right)^{-1}(\mathbf{W}(0-))=(v, p, s)^{T}(0-)$ with $p(0-)=p_{L}(\tau(0-))$. Again (55) is equivalent to (50). Note that the precise value of $s_{0}$ does not matter since the variable $s$ does not appear in the equation for $p_{L}$ nor in the coupling condition.

\section{EXTENSION TO GENERAL LAGRANGIAN SYSTEMS}

We want to extend the coupling to more general systems in Lagrangian coordinates, by transmission of a set of variables which corresponds to $(v, p)$ when restricting to Euler system. We first recall the common algebraic structure of all these systems which allows us to treat their coupling from a general point of view. This is followed by some technical computations which are required in order to define the transmitted variables.

5.1. The form of general Lagrangian systems. We consider systems of $q$ conservation laws in Lagrangian coordinates ( $x$ still stands for a mass variable)

$$
\partial_{t} \mathbf{u}+\partial_{x} \mathbf{f}(\mathbf{u})=\mathbf{0},
$$

which meet some common properties (we refer to [7] for a detailed description):

- They are endowed with a strictly convex entropy $s(\mathbf{u})$, with null associated entropy flux, so that for smooth solutions

$$
\partial_{t} s=0 .
$$

- $\mathbf{u}$ is made of $q-1-d$ state variables and $d$ velocity variables $\mathbf{U}$. The last component of $\mathbf{u}$ is the total energy which we will denote $e$ :

$$
u_{q} \equiv e=\varepsilon+\frac{1}{2}|\mathbf{U}|^{2},
$$

where the internal energy $\varepsilon$ is a state variable. Then $s$ is also a state variable. We will assume that $s(\mathbf{u})$ satisfies $\frac{\partial s}{\partial e}(\mathbf{u}) \equiv s_{e}(\mathbf{u})<0$. The model is then called a fluid model.

- Galilean invariance.

- Reversibility in time for smooth solutions.

Then, such a system can be written in a canonical form: $\exists \Psi: \mathbf{u} \rightarrow \Psi(\mathbf{u}) \in$ $\mathbb{R}^{q-1}, \exists B \in \mathcal{M}\left(\mathbb{R}^{q-1}\right)$ such that

$$
\mathbf{f}(\mathbf{u})=\left(B \Psi(\mathbf{u}),-\frac{1}{2} \Psi(\mathbf{u})^{T} B \Psi(\mathbf{u})\right)^{T},
$$

moreover $B$ is a symmetric $(q-1) \times(q-1)$ constant matrix. Finally the spectrum of $\mathbf{f}^{\prime}(\mathbf{u})$ is symmetric: if $\lambda(\mathbf{u})$ is an eigenvalue, so is $-\lambda(\mathbf{u})$. In the sequel we will write $\lambda$ in order to shorten the notation. Again we refer to [7] for a detailed proof. 
From now on, we assume these results and we derive some of the properties needed in the following computations.

The first consequence of the last result is that there is an even number, say $2 \mathrm{~m}$, of eigenvalues $\lambda_{i} \neq 0$ and we can number the spectrum as follows: $\lambda_{0}=0$ with multiplicity $q-2 m$ and $\lambda_{1}, \cdots, \lambda_{m}<0, \lambda_{m+1}, \cdots, \lambda_{2 m}>0$.

5.1.1. Number of transmitted variables. Since the interface is characteristic, we cannot expect the continuity of $\mathbf{u}$ at the coupling interface. As illustrated by the case of the Euler system, a reduced number of (nonlinear) functions of $\mathbf{u}$ are expected to be continuous. The aim of this section is to provide the material in order to derive the required set of these functions with linear independent gradients which are Riemann invariants associated to the eigenvalue 0.

First note that (57) implies that a solution which is assumed to be discontinuous across $x=0$ satisfies $B[\Psi]=0$, and one could say that $B \Psi$ are transmitted variables. However, $B$ is not inversible as we will see, and we want to 'extract' more explicit information and define independent transmitted variables from these $q-1$ relations, by some change of variables (the analogue of $v, p$ for Euler system). In particular, the number of these transmitted variables depends on the dimension of $\operatorname{ker} B$ and thus on the multiplicity of the eigenvalue 0 of $\mathbf{f}^{\prime}(\mathbf{u})$.

The set of variables $\Psi$ is derived from the polar variables which we now make precise. We will need other sets of variables which we introduce together with some notation. For the set of conservative variables $\mathbf{u}$ and flux $\mathbf{f}(\mathbf{u})$, we distinguish the last component

$$
\mathbf{u}=\left(\mathbf{u}_{q-1}, e\right)^{T}, \mathbf{f}(\mathbf{u})=\left(\mathbf{f}_{q-1}(\mathbf{u}), f_{e}(\mathbf{u})\right)^{T},
$$

where $\mathbf{u}_{q-1}=\left(u_{1}, u_{2}, \cdots, u_{q-1}\right)^{T}$. We should write $\mathbf{u}=\left(\mathbf{u}_{q-1}^{T}, e\right)^{T}$ but we will skip the interior transpose mark $T$ in order to lighten the notation. Thus Després' result says that we can write $\mathbf{f}_{q-1}(\mathbf{u})=B \Psi(\mathbf{u}), f_{e}(\mathbf{u})=-\frac{1}{2} \Psi^{T} B \Psi(\mathbf{u})$ so that the system (56) writes

$$
\left\{\begin{array}{l}
\partial_{t} \mathbf{u}_{q-1}+B \partial_{x} \Psi(\mathbf{u})=\mathbf{0} \\
\partial_{t} e+\partial_{x}\left(-\frac{1}{2} \Psi(\mathbf{u})^{T} B \Psi(\mathbf{u})\right)=0
\end{array}\right.
$$

The system is endowed with an entropy $s$, with $s_{e}(\mathbf{u})<0$, and we define the entropy variables that symmetrize the system (see [1])

$$
\mathbf{u}^{*} \equiv s^{\prime}(\mathbf{u})^{T}=\left(s_{u_{1}}, \cdots, s_{u_{q-1}}, s_{e}\right)^{T}
$$

and (cf. 7] $) \Psi(\mathbf{u})$ in (58) is in fact derived from the entropy variables

$$
\Psi(\mathbf{u})=\left(\frac{s_{u_{1}}}{s_{e}}, \cdots, \frac{s_{u_{q-1}}}{s_{e}}\right)^{T} .
$$

Note that we identify the derivative of scalar functions involved in the definition of polar variables (such as $s^{\prime}(\mathbf{u})$ ) with a $1 \times q$ matrix (line vector). We also consider the change of variables

$$
\mathbf{v}=\left(u_{1}, u_{2}, \cdots, u_{q-1}, s\right)^{T}=\left(\mathbf{u}_{q-1}, s\right)^{T} .
$$

Then, since again $s_{e}(\mathbf{u})<0$, the mapping $\mathbf{v} \rightarrow e(\mathbf{v})=\varepsilon+|\mathbf{U}|^{2} / 2$ is a convex entropy for the system in variables $\mathbf{v}$ (see the details in [1] chapter II, Section 1 for the Euler system) and we define the conjugate function or polar variables by

$$
\mathbf{v}^{*}=e^{\prime}(\mathbf{v})^{T}=\left(e_{v_{1}}, \cdots, e_{v_{q-1}}, e_{s}\right)^{T} \equiv\left(\mathbf{v}_{n-1}^{*}, s^{*}\right)^{T} .
$$


Lemma 1. Let $\mathbf{v}_{n-1}^{*}$ be defined by (60). Then we have the identity

$$
\mathbf{v}_{n-1}^{*}=-\Psi(\mathbf{u}(\mathbf{v}))
$$

where $\Psi$ is defined by (59).

Proof. Indeed, we can write $e(\mathbf{v}(\mathbf{u}))=e=u_{q}$, so that $e^{\prime}(\mathbf{v}) \mathbf{v}^{\prime}(\mathbf{u})=(0, \cdots, 0,1)$. Then, the last component gives $e_{s}=\left(s_{e}\right)^{-1}$ while for the $q-1$ first components, for which $v_{j}=u_{j}$, we get

$$
\sum_{1 \leq i \leq q-1} \frac{\partial e(\mathbf{v})}{\partial v_{i}} \frac{\partial v_{i}}{\partial u_{j}}+\frac{\partial e}{\partial s} \frac{\partial s}{\partial u_{j}}=e_{v_{j}}+e_{s} s_{u_{j}}=0
$$

so that $e_{v_{j}}=-e_{s} s_{u_{j}}=-\left(s_{e}\right)^{-1} s_{u_{j}}$, which, in view of (59), gives (61).

Thus, from (58), we can write for smooth solutions

$$
\partial_{t} \mathbf{v}-\left(\begin{array}{cc}
B & 0 \\
0 & 0
\end{array}\right) \partial_{x} \mathbf{v}^{*}=\mathbf{0}
$$

(in fact, only the last equation is not satisfied by discontinuous solutions). Now multiplying this equation by the matrix $\mathbf{v}^{* \prime}(\mathbf{v})=e^{\prime \prime}(\mathbf{v})$, we get the system satisfied by $\mathbf{v}^{*}$ :

$$
\partial_{t} \mathbf{v}^{*}-e^{\prime \prime}(\mathbf{v})\left(\begin{array}{cc}
B & 0 \\
0 & 0
\end{array}\right) \partial_{x} \mathbf{v}^{*}=\mathbf{0} .
$$

It is not difficult to prove that $\mathbf{u} \rightarrow \mathbf{v}^{*}$ is an admissible change of variables, hence the matrices $-\mathbf{f}^{\prime}(\mathbf{u})$ and $e^{\prime \prime}(\mathbf{v})\left(\begin{array}{cc}B & 0 \\ 0 & 0\end{array}\right)$ are similar. This implies the following result.

Lemma 2. 0 is an eigenvalue of multiplicity $q-2 m-1$ of $B$. Moreover $B$ has $m$ negative and $m$ positive eigenvalues.

Proof. Since $B$ is symmetric, $B$ is diagonalizable. Assume 0 is an eigenvalue of multiplicity $k$ of $B$. Let $\mathbf{r}_{1}, \cdots, \mathbf{r}_{k}$ be $k$ independent eigenvectors $\left(\mathbf{r}_{i} \in \mathbb{R}^{q-1}\right)$ of $B$ associated to the eigenvalue 0 (i.e. a basis of $\operatorname{ker} B$ ) and $\tilde{\mathbf{r}}_{i}=\left(\mathbf{r}_{i}^{T}, 0\right)^{T} \in \mathbb{R}^{q}$. Then the $\tilde{\mathbf{r}}_{i}$ are independent eigenvectors of the augmented matrix

$$
\tilde{B} \equiv\left(\begin{array}{cc}
B & 0 \\
0 & 0
\end{array}\right)
$$

and thus of $e^{\prime \prime}(\mathbf{v}) \tilde{B}$. Now the vector $\mathbf{r}_{q}=(0, \cdots, 0,1)^{T}$ is also an eigenvector of the matrix $\tilde{B}$ associated to 0 and thus of $e^{\prime \prime}(\mathbf{v}) \tilde{B}$ (associated to the eigenvalue 0 ). The $k+1$ vectors $\tilde{\mathbf{r}}_{i}$ and $\mathbf{r}_{q}$ are linearly independent. We have assumed at the beginning that the matrix $\mathbf{f}^{\prime}(\mathbf{u})$ (and thus $e^{\prime \prime}(\mathbf{v}) \tilde{B}$ ) has $q-2 m$ null eigenvalues, hence $k+1 \leq q-2 m$. Let $\mathbf{r}$ be another eigenvector of $e^{\prime \prime}(\mathbf{v}) \tilde{B}$ associated to 0 . Since $e^{\prime \prime}(\mathbf{v})$ is invertible, it is an eigenvector of $\tilde{B}$; we can choose $\tilde{\mathbf{r}} \in \mathbf{r}_{q}^{\perp}$, i.e. $\mathbf{r}=\left(r_{1}, \cdots, r_{q-1}, 0\right)^{T}$. Then, $\left(r_{1}, \cdots, r_{q-1}\right)^{T}$ is clearly an eigenvector of $B$ and thus a combination of the $\mathbf{r}_{i}$, which yields $k+1=q-2 m$.

The diagonalization of the matrix $\mathbf{f}^{\prime}(\mathbf{u})$ gives a diagonal matrix, say $D(\mathbf{u})$, with $m$ negative, $m$ positive and $(q-2 m)$ zero elements, and thus its signature is $(m, m)$. The matrices $\mathbf{f}^{\prime}(\mathbf{u}(\mathbf{v}))$ and $e^{\prime \prime}(\mathbf{v}) \tilde{B}$ are similar. The matrix $e^{\prime \prime}$ is symmetric positive definite. We may define its square root, say $e^{1 / 1 / 2}$, which is symmetric positive definite too, with the inverse $e^{\prime \prime-1 / 2}$. Then $e^{\prime 1 / 2} \tilde{B} e^{\prime 1 / 2}$ is similar to $e^{\prime \prime}(\mathbf{v}) \tilde{B}$. The symmetric matrix $e^{\prime \prime 1 / 2} \tilde{B} e^{\prime \prime 1 / 2}$ is associated to a quadratic form on $\mathbb{R}^{q}$ which thus 
has the same signature $(m, m)$ as $D(\mathbf{u})$. Then $e^{\prime 1 / 2} \tilde{B} e^{\prime 1 / 2}$ has the same signature as $\tilde{B}$. Indeed, the number of eigenvalues that are positive, negative, or 0 does not change under a congruence transformation (by Sylvester's inertial law). Finally if the signature of $\tilde{B}$ is $(m, m)$, so is the signature of $B$. The argument also proves that $\operatorname{dim} \operatorname{ker} B=q-2 m-1$.

5.1.2. Choice of transmitted variables. We are looking for still another set of variables built from $\mathbf{v}^{*}$ for which the $q-1$ first jump relations in the original system or equivalently in system (57): $B\left[\mathbf{v}_{n-1}^{*}\right]=B[\Psi]=0$ will give the conservation of precisely $2 m$ independent quantities, say $\mathbf{w}_{2 m}^{*}$, i.e. $2 m=q-(k+1)$ Riemann invariants associated to the eigenvalue 0 of multiplicity $k+1$,

$$
\left[\mathbf{w}_{2 m}^{*}\right]=\mathbf{0} .
$$

These quantities, corresponding to $v, p$ or rather combinations of $v, p$ for the Euler system, are meant to be transmitted in the coupling which will follow.

More precisely, the aim of this section is to prove that there exists a change of variables $\left(\mathbf{w}_{k}, \mathbf{w}_{2 m}, e\right)$, which will be defined below, such that the weak solutions of the original model (56), (57) equivalently solve the system

$$
\left\{\begin{array}{l}
\partial_{t} \mathbf{w}_{k}=\mathbf{0} \\
\partial_{t} \mathbf{w}_{2 m}-M \partial_{x} \mathbf{w}_{2 m}^{*}=\mathbf{0} \\
\partial_{t} e+\partial_{x}\left(-\frac{1}{2} \mathbf{w}_{2 m}^{*}{ }^{T} M \mathbf{w}_{2 m}^{*}\right)=0
\end{array}\right.
$$

where $M$ is a diagonal invertible matrix and $\mathbf{w}^{*}$ is a linear combination of $\mathbf{w}$. The interest of this change of variables lies in the fact that $\mathbf{w}_{2 m}^{*}$ is the set of $2 m$ Riemann invariants we are looking for. Indeed, the matrix $M$ is constant, diagonal and invertible, hence if a solution is discontinuous across $x=0$ (contact discontinuity corresponding to the eigenvalue $\lambda=0$ ), the jump condition yields

$$
M\left[\mathbf{w}_{2 m}^{*}\right]=\mathbf{0} \Leftrightarrow\left[\mathbf{w}_{2 m}^{*}\right]=\mathbf{0}
$$

and provides the set of transmission relations we are looking for.

We partly 'decouple' the system by diagonalizing $B$. The spectrum $\mathcal{S}$ of $B$ is $\mathcal{S}=\left\{0, \mu_{i}, 1 \leq i \leq 2 m\right\}$ with multiplicity $k$ for the eigenvalue 0 and the other eigenvalues satisfying $\mu_{i} \neq 0$. Since $B \in \mathcal{M}\left(\mathbb{R}^{q-1}\right)$ is symmetric, there exists an orthogonal matrix $O$, satisfying $O O^{T}=I_{q-1}$ and

$$
O B O^{T}=\operatorname{diag}\left(\mathbf{0}_{k}, M\right) \equiv \Lambda,
$$

with $M \in \mathcal{M}\left(\mathbb{R}^{2 m}\right)$ a constant diagonal matrix: $M=\operatorname{diag}\left(\mu_{i}\right)$, having $m$ entries $\mu_{j}<0$ and $m$ entries $\mu_{l}>0$. Then we define the orthogonal matrix $\Omega \in \mathcal{M}\left(\mathbb{R}^{q}\right)$, $\Omega \Omega^{T}=\mathbf{I}_{q}$ by

$$
\Omega=\left(\begin{array}{ll}
O & 0 \\
0 & 1
\end{array}\right)
$$

and set

$$
\mathbf{w}=\Omega \mathbf{v}=\left(\mathbf{w}_{k}, \mathbf{w}_{2 m}, s\right)^{T} .
$$

We adopt the notation: given a vector $\mathbf{y} \in \mathbb{R}^{q}, \mathbf{y}=\left(\mathbf{y}_{1}, \mathbf{y}_{2}, \cdots, \mathbf{y}_{j}\right)^{T}$ corresponds to the partition $\left(i_{1}, i_{2}, \cdots, i_{j}\right)$ of $(1,2, \cdots, q),\left(i_{1}, i_{2}, \cdots, i_{j}\right) \in\left(\mathbb{N}^{*}\right)^{j}, i_{1}+i_{2}+\cdots+i_{j}=q$. 
Also for the particular partition $(k, 2 m, 1),(\mathbf{y})_{2 m} \equiv \mathbf{y}_{2 m}$. With this convention, since $\Omega \tilde{B} \Omega^{T}=\operatorname{diag}\left(\mathbf{0}_{k}, M, 0\right)$, we get from (62) that smooth solutions satisfy

$$
\left\{\begin{array}{l}
\partial_{t} \mathbf{w}_{k}=\mathbf{0} \\
\partial_{t} \mathbf{w}_{2 m}-M \partial_{x}\left(\Omega \mathbf{v}^{*}\right)_{2 m}=\mathbf{0} \\
\partial_{t} s=0
\end{array}\right.
$$

Again, since $\Omega$ has constant entries, only the last equation is not satisfied by discontinuous solutions.

We now introduce

$$
\mathbf{w}^{*}=\Omega \mathbf{v}^{*}=\left(O \mathbf{v}_{n-1}^{*}, s^{*}\right)=\left(\mathbf{w}_{k}^{*}, \mathbf{w}_{2 m}^{*}, s^{*}\right)
$$

so that (66) writes

$$
\left\{\begin{array}{l}
\partial_{t} \mathbf{w}_{k}=\mathbf{0} \\
\partial_{t} \mathbf{w}_{2 m}-M \partial_{x} \mathbf{w}_{2 m}^{*}=\mathbf{0}, \\
\partial_{t} s=0 .
\end{array}\right.
$$

Let us check the following.

Lemma 3. The mapping $\mathbf{w} \rightarrow \mathcal{E}(\mathbf{w}) \equiv e\left(\Omega^{T} \mathbf{w}\right)$ is strictly convex and $\mathcal{E}^{\prime}(\mathbf{w})^{T}=$ $\mathbf{w}^{*}$.

Proof. We use the formula to express the derivative of a compound function. It gives (using the differential form for $e^{\prime \prime}$ )

$$
\mathcal{E}^{\prime \prime}(\mathbf{w})\left(\mathbf{w}_{1}, \mathbf{w}_{2}\right)=e^{\prime \prime}\left(\Omega^{T} \mathbf{w}\right) \cdot\left(\Omega^{T} \mathbf{w}_{1}, \Omega^{T} \mathbf{w}_{2}\right)
$$

or using the matrix form for the Hessian $e^{\prime \prime}(\mathbf{w}), \mathcal{E}^{\prime \prime}(\mathbf{w})=\Omega e^{\prime \prime}\left(\Omega^{T} \mathbf{w}\right) \Omega^{T}$, which proves the convexity of $\mathcal{E}$ since $e^{\prime \prime}(\mathbf{v})$ is positive definite.

Observe that $\mathcal{E}(\mathbf{w}(\mathbf{v}))=e\left(\Omega^{T} \mathbf{w}(\mathbf{v})\right)=e(\mathbf{v})$. Then $\mathcal{E}$ is an entropy for system (68) and the polar variable $\mathcal{E}^{\prime}(\mathbf{w})$ is in fact $\mathbf{w}^{*}=\Omega \mathbf{v}^{*}$, i.e.

$$
\mathcal{E}^{\prime}(\mathbf{w})^{T}=\Omega \mathbf{v}^{*}
$$

(the similarity transformation $\Omega$ commutes with the conjugate). Indeed, for any $\mathbf{h} \in \mathbb{R}^{q}$, the linear form $\mathcal{E}^{\prime}(\mathbf{w})$ satisfies

$$
\mathcal{E}^{\prime}(\mathbf{w}) \cdot \mathbf{h}=e^{\prime}\left(\Omega^{T} \mathbf{w}\right) \cdot \Omega^{T} \mathbf{h}
$$

and if we identify both $\mathcal{E}^{\prime}(\mathbf{w})$ and $e^{\prime}(\mathbf{v})$ with line vectors in $\mathbb{R}^{q}$, it gives

$$
\mathcal{E}^{\prime}(\mathbf{w})^{T}=\Omega e^{\prime}\left(\Omega^{T} \mathbf{w}\right)=\Omega e^{\prime}(\mathbf{v})^{T}=\Omega \mathbf{v}^{*} .
$$

Let us now consider the system satisfied by the set of variables $\mathbf{w}^{e}=\left(\mathbf{w}_{q-1}, e\right)^{T}$, replacing the equation on the entropy $s$ by the original equation in $e$. It can be checked that $\mathbf{w}^{e}$ is also an admissible change of variables and smooth solutions of (66) Solve the energy equation. In order to express the flux in terms of $\mathbf{w}^{e}$, we notice that since $\mathbf{v}^{*}=\Omega^{T} \mathbf{w}^{*}$ and $\Psi^{T} B \Psi=\mathbf{v}_{q-1}^{*}{ }^{T} O^{T} \Lambda O \mathbf{v}_{q-1}^{*}$, we get $\Psi^{T} B \Psi=$ $\mathbf{w}_{q-1}^{*} \Lambda \mathbf{w}_{q-1}^{*}=\mathbf{w}_{2 m}^{*}{ }^{T} M \mathbf{w}_{2 m}^{*}$ because of the specific form of $\Lambda=\operatorname{diag}\left(\mathbf{0}_{k}, M\right)$. Thus, in variables

$$
\mathbf{w}^{e} \equiv\left(\mathbf{w}_{q-1}, e\right)^{T}=\left(\mathbf{w}_{k}, \mathbf{w}_{2 m}, e\right)^{T},
$$


we get as expected the system (64)

$$
\left\{\begin{array}{l}
\partial_{t} \mathbf{w}_{k}=\mathbf{0} \\
\partial_{t} \mathbf{w}_{2 m}-M \partial_{x} \mathbf{w}_{2 m}^{*}=\mathbf{0} \\
\partial_{t} e+\partial_{x}\left(-\frac{1}{2} \mathbf{w}_{2 m}^{*}{ }^{T} M \mathbf{w}_{2 m}^{*}\right)=0
\end{array}\right.
$$

System (64) is now equivalent to the initial one (they have the same smooth and discontinuous solutions) since the last equation is now also satisfied by discontinuous solutions of (56), (57). Also, $\mathbf{w}_{2 m}^{*}$ is indeed the set of $2 m$ Riemann invariants we are looking for, while the $k$ components of $\mathbf{w}_{k}$ and $s$ are common Riemann invariants for the other characteristic fields $\lambda_{j} \neq 0$.

5.1.3. Choice of coupling variables. The 'final' set of variables we are going to use for coupling is

$$
\mathbf{z}=\left(\mathbf{w}_{k}, \mathbf{w}_{2 m}^{*}, e\right)^{T} .
$$

Let us first check that it is indeed admissible. The results of the previous section prove that it is sufficient to ask for the transmission of $\mathbf{w}_{2 m}^{*}$ since both $\mathbf{w}_{k}$ and $e$ are free of constraints at the interface.

Lemma 4. The mapping $\mathbf{w} \rightarrow \mathbf{z}$ defines an admissible change of variables.

Proof. Recall that $\mathbf{w}, \mathbf{z}$ are defined by $\mathbf{w}=\left(\mathbf{w}_{k}, \mathbf{w}_{2 m}, s\right)^{T}$ and $\mathbf{z}=\left(\mathbf{w}_{k}, \mathbf{w}_{2 m}^{*}, e\right)^{T}$, with $e=\mathcal{E}(\mathbf{w})$ strictly convex. Hence it is enough to prove that the $2 m \times 2 m$ matrix $\nabla_{\mathbf{w}_{2 m}} \mathbf{w}_{2 m}^{*} \equiv\left(\left(\partial_{w_{2 m, i}} w_{2 m, j}^{*}\right)_{i, j}\right)$ is invertible. We have $\mathbf{w}^{*}=\mathcal{E}^{\prime}(\mathbf{w})^{T}$, thus $\left(\mathbf{w}^{*}\right)^{\prime}(\mathbf{w})=\mathcal{E}^{\prime \prime}(\mathbf{w})$. Let us write $\mathcal{E}^{\prime \prime}(\mathbf{w})$ in blocks corresponding to the the partition $(k, 2 m, 1)$, i.e. to the decomposition of $\mathbb{R}^{q}$ in $\mathbb{R}^{k} \times \mathbb{R}^{2 m} \times \mathbb{R}$ :

$$
\mathcal{E}^{\prime \prime}(\mathbf{w})=\left(\begin{array}{ccc}
E_{k, k} & E_{k, 2 m} & E_{k, 1} \\
E_{2 m, k} & E_{2 m, 2 m} & E_{2 m, 1} \\
E_{1, k} & E_{1,2 m} & E_{1,1}
\end{array}\right) .
$$

The matrix corresponding to $\nabla_{\mathbf{w}_{2 m}} \mathbf{w}_{2 m}^{*}$ is the $2 m \times 2 m$ diagonal block $E_{2 m, 2 m}$ extracted from $\mathcal{E}^{\prime \prime}(\mathbf{w})$ which is symmetric positive definite. This means that $E_{2 m, 2 m}(\mathbf{w})$ is the restriction of $\mathcal{E}^{\prime \prime}(\mathbf{w})$ to the subspace $\left\{\mathbf{r}=\left(\mathbf{0}_{k}, \mathbf{r}_{2 m}, 0\right), \mathbf{r}_{2 m} \in \mathbb{R}^{2 m}\right\}$ and thus is invertible.

Example. Let us make explicit the above computations for the Euler system, $\mathbf{u}=(\tau, v, e)^{T}, \mathbf{v}=(\tau, v, s)^{T}, s_{e}=\frac{1}{T}, \mathbf{u}^{*}=\frac{1}{T}(p,-v, 1)^{T}, \Psi=(p,-v)^{T}, \mathbf{v}^{*}=$ $(-p, v, T)^{T}, k=0, m=1$. Then

$$
B=\left(\begin{array}{ll}
0 & 1 \\
1 & 0
\end{array}\right), M=\left(\begin{array}{cc}
-1 & 0 \\
0 & 1
\end{array}\right), O=\frac{1}{\sqrt{2}}\left(\begin{array}{cc}
-1 & 1 \\
1 & 1
\end{array}\right),
$$

so that $\mathbf{w}=\Omega \mathbf{v}=\left(\frac{1}{\sqrt{2}}(-\tau+v), \frac{1}{\sqrt{2}}(\tau+v), s\right)^{T}$ and the components of $\mathbf{w}_{2}^{*}=-O \Psi=$ $\frac{1}{\sqrt{2}}(v+p, v-p)^{T}$ are indeed 0-Riemann invariants. The matrix $E_{2 m, 2 m}$ which is the first $2 \times 2$ diagonal block $O^{T} e^{\prime \prime}(\mathbf{v})_{2,2} O$, extracted from $\mathcal{E}^{\prime \prime}(\mathbf{w})=\Omega^{T} e^{\prime \prime}(\mathbf{v}) \Omega$, is given by

$$
E_{2 m, 2 m}=\frac{1}{2}\left(\begin{array}{ll}
1+C^{2} & 1-C^{2} \\
1-C^{2} & 1+C^{2}
\end{array}\right)
$$

Indeed, $e^{\prime}(\mathbf{v})^{T}=\mathbf{v}^{*}=(-p, v, T)^{T}$ so that

$$
e^{\prime \prime}(\mathbf{v})_{2,2}=\left(\begin{array}{cc}
-\partial_{\tau} p & 0 \\
0 & 1
\end{array}\right)
$$

with $-\partial_{\tau} p(\tau, s)=C^{2}$. 
5.2. The coupling of general Lagrangian systems. We now consider the coupling of two general systems (11), (2) where the fluxes $\mathbf{f}_{\alpha}$ are of the form (57) with the same matrix $B$ but with two distinct entropy functions involved in the definition (59) of $\Psi$.

Thus, following the previous study, we start from two systems which we can write in the equivalent form (64) with the set (69) of variables $\mathbf{w}^{e}=\left(\mathbf{w}_{q-1}, e\right)^{T}$, where each system is endowed with a strictly convex entropy function: $s_{L}=s_{L}\left(\mathbf{w}^{e}\right)$, $s_{R}=s_{R}\left(\mathbf{w}^{e}\right)$, and we have $e=\frac{|U|^{2}}{2}+\varepsilon$ with $(\tau, s) \rightarrow \varepsilon(\tau, s)$ strictly convex. We want to express the coupling condition in the set (70) of variables $\mathbf{z}=\left(\mathbf{w}_{k}, \mathbf{w}_{2 m}^{*}, e\right)^{T}$ which means that we want to transmit $\mathbf{w}_{2 m}^{*}$. Note that, considered as a function of $\mathbf{z}, \mathbf{w}_{2 m}^{*}$ depends on the choice of the closure relation.

5.2.1. The coupling conditions expressed in variables $\mathbf{z}$. Recalling (7), we write

$$
\left\{\begin{array}{l}
\mathbf{w}^{e}(0-, t) \in \mathcal{O}_{L}\left(\varphi_{L}(\mathbf{z}(0+, t))\right), \\
\mathbf{w}^{e}(0+, t) \in \mathcal{O}_{R}\left(\varphi_{R}(\mathbf{z}(0-, t))\right),
\end{array}\right.
$$

where $\mathbf{w}^{e}=\varphi_{L}(\mathbf{z})$ in $x<0$, and $\mathbf{w}^{e}=\varphi_{R}(\mathbf{z})$ in $x>0$. We aim to prove that (71) gives the continuity of $\mathbf{w}_{2 m}^{*}$ (for the Euler system; this means the continuity of $v$ and $p$ ) at the interface.

This result is stated in the following proposition. It will be established for entropy functions close enough so that the following assumption is true: given, for $\alpha=L, R$, a basis of eigenvectors $\left(\mathbf{r}_{j}^{\alpha}\right)_{1 \leq j \leq q}$ of the Jacobian matrix $\mathbf{f}_{\alpha}^{\prime}$ and $\left(\mathbf{r}_{j, 2 m}^{\alpha}\right)_{1 \leq j \leq 2 m}$ the corresponding basis of $\mathbb{R}^{2 m}$, the vectors $\left(\mathbf{r}_{1,2 m}^{R}, \mathbf{r}_{2,2 m}^{R}, \cdots, \mathbf{r}_{m, 2 m}^{R}, \mathbf{r}_{m+1,2 m}^{L}, \mathbf{r}_{m+2,2 m}^{L}\right.$, $\cdots, \mathbf{r}_{2 m, 2 m}^{L}$ ) still form a basis of $\mathbb{R}^{2 m}$. The vectors will in fact be expressed as functions of $\mathbf{w}^{e}$.

Proposition 7. The coupling conditions (71) lead to the continuity of $\mathbf{w}_{2 m}^{*}$ at the interface $x=0$.

The proof relies on some more technical lemmas.

Lemma 5. Given a state $\mathbf{z}$, let $\mathbf{C}_{L}^{+}(\mathbf{z})$ be defined as the projection (on the $\mathbf{w}_{2 m}^{*}$ hyperplane) of the set of states that can be connected to $\varphi_{L}(\mathbf{z})$ by (at most) $m$ $L$-waves associated to positive eigenvalues $\lambda_{j}^{L}, j \in\{m+1, \cdots, 2 m\}$, and similarly $\mathbf{C}_{R}^{-}(\mathbf{z})$ as the projection of the set of states to which $\varphi_{R}(\mathbf{z})$ can be connected by (at most) $m R$-waves associated to negative eigenvalues $\lambda_{j}^{R}, j \in\{1, \cdots, m\}$. Then (71) implies

$$
\mathbf{w}_{2 m}^{*}(0-, t) \in \mathbf{C}_{L}^{+}(\mathbf{z}(0+, t)), \mathbf{w}_{2 m}^{*}(0+, t) \in \mathbf{C}_{R}^{-}(\mathbf{z}(0-, t)) .
$$

Proof. By definition of the admissible set $\mathcal{O}_{L}$, there exists a state $\mathbf{w}_{-}^{e} \in \Omega$ such that

$$
\mathbf{w}^{e}(0-, t)=\mathbf{W}_{L}\left(0-; \mathbf{w}_{-}^{e}, \varphi_{L}(\mathbf{z}(0+, t))\right) .
$$

The $L-$ Riemann problem between $\mathbf{w}_{-}^{e}$ and $\varphi_{L}(\mathbf{z}(0+, t))$ is thus built with a succession of (at most) $m L$-waves (with negative speed) between $\mathbf{w}_{-}^{e}$ and $\mathbf{w}^{e}(0-, t)$, a 0 -contact discontinuity at $x=0$ between $\mathbf{w}^{e}(0-, t)$ and a state $\mathbf{w}_{+}^{e, L}$ and (at most) $m L$-waves (with positive speed) between $\mathbf{w}_{+}^{e, L}$ and $\varphi_{L}(\mathbf{z}(0+, t))$. This yields that

$$
\mathbf{w}_{2 m}^{*}\left(\mathbf{w}_{+}^{e, L}\right)=\mathbf{w}_{2 m}^{*}(0-, t),
$$

and thus, after projection on $\mathbb{R}^{2 m}$ (the $\mathbf{w}_{2 m}^{*}$-hyperplane), $\mathbf{w}_{2 m}^{*}(0-, t)$ belongs to the set $\mathbf{C}_{L}^{+}(\mathbf{z}(0+, t))$, defined as the projection (on the $\mathbf{w}_{2 m}^{*}$-hyperplane) of the set 
of states that can be connected to $\varphi_{L}(\mathbf{z}(0+, t))$ by $L$-waves associated to positive eigenvalues $\lambda_{j}^{L}, j \in\{m+1, \cdots, 2 m\}$.

Similarly by definition of the admissible set $\mathcal{O}_{R}$, there exists a state $\mathbf{w}_{+}^{e} \in \Omega$ such that

$$
\mathbf{w}^{e}(0+, t)=\mathbf{W}_{R}\left(0+; \varphi_{R}(\mathbf{z}(0-, t)), \mathbf{w}_{+}^{e}\right) .
$$

The $R$-Riemann problem between $\varphi_{R}(\mathbf{z}(0-, t))$ and $\mathbf{w}_{+}^{e}$ is thus built with a succession of (at most) $m R$-waves between $\varphi_{R}(\mathbf{z}(0-, t))$ and a state $\mathbf{w}_{-}^{e, R}$, a 0 -contact discontinuity at $x=0$ between $\mathbf{w}_{-}^{e, R}$ and $\mathbf{w}^{e}(0+, t)$ and (at most) $m R$-waves between $\mathbf{w}^{e}(0+, t)$ and $\mathbf{w}_{+}^{e}$. This yields that

$$
\mathbf{w}_{2 m}^{*}\left(\mathbf{w}_{-}^{e, R}\right)=\mathbf{w}_{2 m}^{*}(0+, t),
$$

and thus, after projection on $\mathbb{R}^{2 m}$ (the $\mathbf{w}_{2 m}^{*}$-hyperplane), $\mathbf{w}_{2 m}^{*}(0+, t)$ belongs to the set $\mathbf{C}_{R}^{-}(\mathbf{z}(0-, t))$ defined as the projection of the set of states to which $\varphi_{R}(\mathbf{z}(0-, t))$ can be connected by $R$-waves associated to negative eigenvalues $\lambda_{j}^{R}$, $j \in\{1, \cdots, m\}$.

However, the proof of the continuity of $\mathbf{w}_{2 m}^{*}$ at 0 supposes that we can parametrize correctly the projection of the wave curves $\mathbf{C}_{R}^{-}(\mathbf{z}(0-, t))$ or $\mathbf{C}_{L}^{+}(\mathbf{z}(0+, t))$, at least locally.

For instance, for the Euler system, we are able to parametrize the projection of the wave curves in the $(v, p)$-plane in the form $v=\Phi(p)$. The parametization is proved in the following lemma where we use the same notation as that introduced in Lemma 5.

Lemma 6. For given states $\mathbf{z}^{ \pm}$characterized by $\mathbf{z}^{ \pm}=\left(\mathbf{w}_{k}^{ \pm}, \mathbf{w}_{2 m}^{*}{ }^{ \pm}, e^{ \pm}\right)^{T}$, the curve $\mathbf{C}_{R}^{-}\left(\mathbf{z}^{-}\right)$can be parametrized for $\xi^{-}=\left(\xi_{j}^{-}\right) \in \mathbb{R}^{m},\left|\xi_{j}^{-}\right|$small enough by

$$
\mathbf{w}_{2 m}^{*}\left(\xi^{-}\right)=\mathbf{w}_{2 m}^{*}-+\sum_{j=1}^{m} \xi_{j}^{-} \mathbf{r}_{j, 2 m}^{R}\left(\mathbf{z}^{-}\right)+O\left(\left|\xi^{-}\right|^{2}\right) ;
$$

similarly $\mathbf{C}_{L}^{+}\left(\mathbf{z}^{+}\right)$can be parametrized for $\xi^{+}=\left(\xi_{j}^{+}\right) \in \mathbb{R}^{m},\left|\xi_{j}^{+}\right|$small enough, by

$$
\mathbf{w}_{2 m}^{*}\left(\xi^{+}\right)=\mathbf{w}_{2 m}^{*}+\sum_{j=m+1}^{2 m} \xi_{j}^{+} \mathbf{r}_{j, 2 m}^{L}\left(\mathbf{z}^{+}\right)+O\left(\left|\xi^{+}\right|^{2}\right) \text {. }
$$

Proof. Let us first consider a discontinuous solution of (64). It satisfies the following jump conditions:

$$
\left\{\begin{array}{l}
-\sigma\left[\mathbf{w}_{k}\right]=\mathbf{0} \\
-\sigma\left[\mathbf{w}_{2 m}\right]-M\left[\mathbf{w}_{2 m}^{*}\right]=\mathbf{0} \\
-\sigma[e]-\frac{1}{2}\left[\mathbf{w}_{2 m}^{*}{ }^{T} M \mathbf{w}_{2 m}^{*}\right]=0
\end{array}\right.
$$

in particular $\left[\mathbf{w}_{2 m}^{*}\right]=-\sigma M^{-1}\left[\mathbf{w}_{2 m}\right]$ with $M$ diagonal.

A shock necessarily corresponds to a $j$-characteristic field associated to a non null eigenvalue $\lambda_{j}, j \in\{1, \cdots, 2 m\}$. Assume first, only in order to simplify the presentation, that all the corresponding fields are genuinely nonlinear (GNL). We know that the jump $\left[\mathbf{w}_{e}\right]$ is an eigenvector, say $\mathbf{r}_{j}^{e}$, of the matrix noted in a shortened way $\mathbf{D}^{e}$ :

$$
\mathbf{D}^{e} \equiv \mathbf{D}\left(\mathbf{w}_{-}^{e}, \mathbf{w}_{+}^{e}\right)=\int_{0}^{1} \mathbf{D}\left(\mathbf{w}_{-}^{e}+s\left(\mathbf{w}_{+}^{e}-\mathbf{w}_{-}^{e}\right)\right) d s
$$

corresponding to the eigenvalue $\sigma$. 
Note that the choice of variables $\mathbf{z}$ rather decouples the system in three subsystems,

$$
\left\{\begin{array}{l}
\partial_{t} \mathbf{w}_{k}=\mathbf{0}, \\
\partial_{t} \mathbf{w}_{2 m}^{*}-E_{2 m, 2 m} M \partial_{x} \mathbf{w}_{2 m}^{*}=\mathbf{0}, \\
\partial_{t} e+\partial_{x}\left(-\frac{1}{2} \mathbf{w}_{2 m}^{*}{ }^{T} M \mathbf{w}_{2 m}^{*}\right)=0 .
\end{array}\right.
$$

The matrix $\mathbf{D}^{*}(\mathbf{z})$ of this quasilinear system $\partial_{t} \mathbf{z}+\mathbf{D}^{*}(\mathbf{z}) \partial_{x} \mathbf{z}=0$ is (in blocks of sizes corresponding to the decomposition $q=k+2 m+1$ )

$$
\mathbf{D}^{*}(\mathbf{z})=\left(\begin{array}{ccc}
0 & 0 & 0 \\
0 & -E_{2 m, 2 m} M & 0 \\
0 & B_{1,2 m} & 0
\end{array}\right) .
$$

The Jacobian matrix of system (64), noted $\mathbf{D}$, is similar to $\mathbf{D}^{*}$ and has the same structure in blocks as $\mathbf{D}^{*}(\mathbf{z})$ :

$$
\mathbf{D}\left(\mathbf{w}^{e}\right)=\left(\begin{array}{ccc}
0 & 0 & 0 \\
0 & -M E_{2 m, 2 m} & 0 \\
0 & B_{1,2 m} E_{2 m, 2 m} & 0
\end{array}\right) .
$$

Hence, given the structure of $\mathbf{D}\left(\mathbf{w}^{e}\right)$ (see (75)), this eigenvector $\mathbf{r}_{j}^{e}$ of $\mathbf{D}^{e}$ has the form $\mathbf{r}_{j}^{e}=\left(\mathbf{0}_{k}, \mathbf{r}_{j, 2 m}^{e}, r_{j, e}\right)^{T}$. Now, by (74) and (75)), the matrices extracted from $\mathbf{D}$ and $\mathbf{D}^{*}$ are given by $\mathbf{D}_{2 m, 2 m}^{*}=-E_{2 m, 2 m} M$ and $\mathbf{D}_{2 m, 2 m}=-M E_{2 m, 2 m}$. We write

$$
\left[\mathbf{w}_{2 m}^{*}\right]=-\sigma M^{-1}\left[\mathbf{w}_{2 m}\right]=-M^{-1} \mathbf{D}_{2 m, 2 m}^{e} \mathbf{r}_{j, 2 m}^{e}=E_{2 m, 2 m}^{e} \mathbf{r}_{j, 2 m}^{e}=\mathbf{r}_{j, 2 m}^{z}
$$

with shorthand notation expressing that $\mathbf{r}_{j, 2 m}^{z} \equiv E_{2 m, 2 m}^{e} \mathbf{r}_{j, 2 m}^{e}$ is an eigenvector of $\mathbf{D}_{2 m, 2 m}^{*}$.

Thus we can parametrize the $j$-shock curve in a decoupled way. For a given left state characterized by $\mathbf{z}^{-}=\left(\mathbf{w}_{k}^{-}, \mathbf{w}_{2 m}^{*}{ }^{-}, e^{-}\right)^{T}$, the curve (the $j$-shock curve) of states which can be connected to $\mathbf{z}^{-}$by a $j$-shock can be parametrized in variable $\mathbf{z}$, and for $|\xi|$, small enough $\xi<0$ (this results from Lax entropy condition) and we may write

$$
\left\{\begin{array}{l}
\mathbf{w}_{k}(\xi)=\mathbf{w}_{k}^{-}, \\
\mathbf{w}_{2 m}^{*}(\xi)=\mathbf{w}_{2 m}^{*}{ }^{-}+\xi \mathbf{r}_{j, 2 m}\left(\mathbf{z}^{-}\right)+O\left(\xi^{2}\right)
\end{array}\right.
$$

and the last equation of (172) which we write

$$
e(\xi)=e^{-}+\frac{1}{2 \sigma}\left[\mathbf{w}_{2 m}^{*}{ }^{T} M \mathbf{w}_{2 m}^{*}\right]
$$

Now, a $j$-rarefaction curve, where the index $j$ corresponds again to a nonnull eigenvalue $\lambda_{j}, j \in\{1, \cdots, 2 m\}$, is (in variable $\mathbf{z}$ ) an integral curve of $\mathbf{r}_{j}(\mathbf{z})$ and thus satisfies

$$
\left\{\begin{array}{l}
d_{\xi} \mathbf{w}_{k}=\mathbf{0} \\
d_{\xi} \mathbf{w}_{2 m}^{*}=\mathbf{r}_{j, 2 m}(\mathbf{z}(\xi))
\end{array}\right.
$$

together with

$$
d_{\xi} s=0 .
$$

For a given left state $\mathbf{z}^{-}=\left(\mathbf{w}_{k}^{-}, \mathbf{w}_{2 m}^{*}{ }^{-}, e^{-}\right)^{T}$, the curve of states which can be connected to $\mathbf{z}^{-}$by a $j$-rarefaction can be parametrized for $\xi>0$ small enough by

$$
\left\{\begin{array}{l}
\mathbf{w}_{k}(\xi)=\mathbf{w}_{k}^{-}, \\
\mathbf{w}_{2 m}^{*}\left(\xi^{-}\right)=\mathbf{w}_{2 m}^{*}{ }^{-}+\xi \mathbf{r}_{j, 2 m}\left(\mathbf{z}^{-}\right)+O\left(\xi^{2}\right), \\
e(\xi)=\mathcal{E}\left(\mathbf{w}_{k}^{-}, \mathbf{w}_{2 m}^{*}(\xi), s^{-}\right) .
\end{array}\right.
$$


$\mathcal{E}(\mathbf{w})$ is a function of $\mathbf{w}=\left(\mathbf{w}_{k}, \mathbf{w}_{2 m}, s\right)^{T}$, and we have seen that $\nabla_{\mathbf{w}_{2 m}} \mathbf{w}_{2 m}^{*}$ is invertible; hence the notation $\mathcal{E}\left(\mathbf{w}_{k}, \mathbf{w}_{2 m}^{*}, s\right)$ is a short way of writing $\mathcal{E}\left(\mathbf{w}_{k}, \mathbf{w}_{2 m}\left(\mathbf{w}_{2 m}^{*}\right), s\right)$.

Now if a characteristic field, say $\lambda_{j}^{R}$, is linearly degenerate (LD), the result still holds since the curve of states which can be connected to $\mathbf{z}^{-}$by a $j$-contact discontinuity is also an integral curve of $\mathbf{r}_{j}(\mathbf{z})$ that can be parametrized in the same way.

Thus, for a given left state $\mathbf{z}^{-}=\left(\mathbf{w}_{k}^{-}, \mathbf{w}_{2 m}^{*}{ }^{-}, e^{-}\right)$, the curve $\mathbf{C}_{R}^{-}\left(\mathbf{z}^{-}\right)$projection of the set of states to which $\varphi_{L}\left(\mathbf{z}^{-}\right)$can be connected by (at most) $m R$-waves, $j$-rarefaction, $j$-shock (if the $j$-field is GNL) or $j$-contact discontinuity (if it is LD), each associated to a negative eigenvalue $\lambda_{j}^{R}, j \in\{1, \cdots, m\}$, can be parametrized for $\xi^{-}=\left(\xi_{j}^{-}\right) \in \mathbb{R}^{m},\left|\xi_{j}^{-}\right|$small enough by

$$
\mathbf{w}_{2 m}^{*}\left(\xi^{-}\right)=\mathbf{w}_{2 m}^{*}{ }^{-}+\sum_{j=1}^{m} \xi_{j}^{-} \mathbf{r}_{j, 2 m}^{R}\left(\mathbf{z}^{-}\right)+O\left(\left|\xi^{-}\right|^{2}\right) .
$$

Similarly, for $\mathbf{C}_{L}^{+}\left(\mathbf{z}^{+}\right)$the projection of the set of states to which $\varphi_{L}\left(\mathbf{z}^{+}\right)$can be connected by (at most) $m L$-waves, $j$-rarefaction or $j$-shock or $j$-contact discontinuity, each associated to a positive eigenvalue $\lambda_{j}^{L}, j \in\{1, \cdots, m\}$, can be parametrized for $\xi^{+}=\left(\xi_{j}^{+}\right) \in \mathbb{R}^{m},\left|\xi_{j}^{+}\right|$small enough, by

$$
\mathbf{w}_{2 m}^{*}\left(\xi^{+}\right)=\mathbf{w}_{2 m}^{*}+\sum_{j=m+1}^{2 m} \xi_{j}^{+} \mathbf{r}_{j, 2 m}^{L}\left(\mathbf{z}^{+}\right)+O\left(\left|\xi^{+}\right|^{2}\right),
$$

which ends the proof of the lemma.

Proof of Proposition 7. We apply the above results to $\mathbf{z}^{+}=\mathbf{z}(0+, t), \mathbf{z}^{-}=\mathbf{z}(0-, t)$, and assume that $\mathbf{r}_{j, 2 m}^{R}(\mathbf{z}(0+, t)), 1 \leq j \leq m, \mathbf{r}_{j, 2 m}^{L}(\mathbf{z}(0-, t)), m+1 \leq j \leq 2 m$, are linearly independent. We write

$$
\left\{\begin{array}{l}
\mathbf{w}_{2 m}^{*}(0+, t)=\mathbf{w}_{2 m}^{*}(0-, t)+\sum_{j=1}^{m} \xi_{j}^{-} \mathbf{r}_{j, 2 m}^{R}(\mathbf{z}(0-, t))+O\left(\left|\xi^{-}\right|^{2}\right), \\
\mathbf{w}_{2 m}^{*}(0-, t)=\mathbf{w}_{2 m}^{*}(0+, t)+\sum_{j=m+1}^{2 m} \xi_{j}^{+} \mathbf{r}_{j, 2 m}^{L}(\mathbf{z}(0+, t))+O\left(\left|\xi^{+}\right|^{2}\right) .
\end{array}\right.
$$

Assume $\xi=\left(\xi^{-}, \xi^{+}\right)$is nonnull. This would imply

$$
\sum_{j=1}^{m} \frac{\xi_{j}^{-}}{|\xi|} \mathbf{r}_{j, 2 m}^{R}(\mathbf{z}(0-, t))=-\sum_{j=m+1}^{2 m} \frac{\xi_{j}^{+}}{|\xi|} \mathbf{r}_{j, 2 m}^{L}(\mathbf{z}(0+, t))+O(|\xi|) .
$$

This holds for any $\xi \neq 0$ small enough, letting $\xi$ tend to 0 . This yields the fact that some of the vectors $\mathbf{r}_{j, 2 m}^{R}, 1 \leq j \leq m$, and $\mathbf{r}_{j, 2 m}^{L}, m+1 \leq j \leq 2 m$, are linearly dependent, in contradiction with our assumption. Hence $\xi=0$ and $\mathbf{w}_{2 m}^{*}(0-, t)=$ $\mathbf{w}_{2 m}^{*}(0+, t)$, which concludes the proof.

The fact that the vectors $\mathbf{r}_{j, 2 m}^{R}(\mathbf{z}(0+, t)), 1 \leq j \leq m, \mathbf{r}_{j, 2 m}^{L}(\mathbf{z}(0-, t)), m+1 \leq j \leq$ $2 m$, are linearly independent can be proved, by some technical continuity argument, using the fact that we have assumed that the entropy laws are close enough to ensure that the eigenvectors $\mathbf{r}_{j, 2 m}^{R}(\mathbf{z}), 1 \leq j \leq m, \mathbf{r}_{j, 2 m}^{L}(\mathbf{z}), m+1 \leq j \leq 2 m$, are independent. Indeed, due to the coupling condition, we know that $\mathbf{z}(0-, t)$ and $\mathbf{z}(0+, t)$ are connected by $L-$ (or equivalently $R-$ ) waves, hence, with a possible change in $O\left(\left|\xi^{-}\right|^{2}\right)$ we can take all the eigenvectors evaluated at the same state $\mathbf{z}(0+, t)($ or $\mathbf{z}(0-, t))$. 
5.2.2. The coupled Riemann problem. We are given two nearby constant states $\mathbf{w}_{ \pm}^{e}$ or equivalently $\mathbf{z}_{ \pm}=\left(\mathbf{w}_{k}^{ \pm}, \mathbf{w}_{2 m}^{*}{ }^{ \pm}, e^{ \pm}\right)$, where $\mathbf{w}_{-}^{e}=\varphi_{L}\left(\mathbf{z}_{-}\right), \mathbf{w}_{+}^{e}=\varphi_{R}\left(\mathbf{z}_{+}\right)$, and we want to solve the coupled Riemann problem, i.e. (64) with $s_{L}=s_{L}\left(\mathbf{w}^{e}\right)$, or in variable $\mathbf{z}, \varepsilon_{L}(\tau, s)$ in $x<0, s_{R}=s_{R}\left(\mathbf{w}^{e}\right)$ or $\varepsilon_{R}(\tau, s)$ in $x>0$, the initial condition

$$
\mathbf{w}(x, 0)=\left\{\begin{array}{l}
\mathbf{w}_{-}^{e}, \text { in } x<0, \\
\mathbf{w}_{+}^{e}, \text { in } x>0,
\end{array}\right.
$$

together with the coupling conditions (71).

Theorem 1. Assuming the above hypothesis, the coupled Riemann problem has a unique solution.

Proof. We try to connect the states by a succession of elementary waves: (at most) $m L$-waves, each associated to a negative eigenvalue $\lambda_{j}^{L}<0, j \in\{1, \cdots, m\}$, between $\mathbf{w}_{-}^{e}$ and $\mathbf{w}^{e}(0-)$, a 'discontinuity' at the interface $x=0$ between $\mathbf{w}^{e}(0-)$ and $\mathbf{w}^{e}(0+)$ satisfying the coupling conditions (71) and (at most) $m R$-waves, each associated to a positive eigenvalue $\lambda_{j}^{R}>0, j \in\{m+1, \cdots, 2 m\}$, between $\mathbf{w}^{e}(0+)$ and $\mathbf{w}_{+}^{e}$.

Following the proof of Proposition 7, we intend to 'project' on the $\mathbf{w}_{2 m}^{*}$ hyperplane since the discontinutity between the states $\mathbf{w}^{e}(0-)$ and $\mathbf{w}^{e}(0+)$ is characterized by $\mathbf{w}_{2 m}^{*}(0-)=\mathbf{w}_{2 m}^{*}(0+)$. This gives $2 m$ unknown quantities $\left(\xi_{j}\right)_{1 \leq j \leq 2 m}$ characterizing the components $\mathbf{w}_{2 m}^{*}$ of the intermediate constant states, say $\mathbf{w}_{j, *}^{e}$, between the $L$-waves (in $x<0$ ) or $R$-waves (in $x>0$ ). These quantities are obtained by writing the $2 m$ equations expressing that $\mathbf{w}_{j, *}^{e}$ belongs to the $j$-wave curve through $\mathbf{w}_{j-1, *}^{e}$ or $\mathbf{w}_{j+1, *}^{e}$ according to whether $j$ is in $\{1, \cdots, m\}$ or $\{m+1, \cdots, 2 m\}$. Using the parametrization of Lemma 6 , it results in

$$
\left\{\begin{array}{l}
\mathbf{w}_{2 m}^{*}(0-, t)=\left(\mathbf{w}_{2 m}^{*}\right)^{-}+\sum_{j=1}^{m} \xi_{j} \mathbf{r}_{j, 2 m}^{L}(\mathbf{z}(0-, t))+0\left(\left|\xi^{+}\right|^{2}\right), \\
\mathbf{w}_{2 m}^{*}(0+, t)=\left(\mathbf{w}_{2 m}^{*}\right)^{+}+\sum_{j=m+1}^{2 m} \xi_{j} \mathbf{r}_{j, 2 m}^{R}(\mathbf{z}(0+, t))+0\left(\left|\xi^{-}\right|^{2}\right) .
\end{array}\right.
$$

Now, since the $2 m$ vectors $\mathbf{r}_{j, 2 m}^{L}(\mathbf{z}(0-, t)), \mathbf{r}_{j, 2 m}^{R}(\mathbf{z}(0+, t))$ are independent, thanks to the inverse mapping theorem, we conclude that the $\xi_{j}$ exist and are unique for $\mathbf{z}^{ \pm}$sufficiently close.

We already know that the $\mathbf{w}_{k}$ are constant across the non- 0 characteristic fields; thus $\mathbf{w}_{k}(0-)=\mathbf{w}_{k}^{-}, \mathbf{w}_{k}(0+)=\mathbf{w}_{k}^{+}$. If there were only rarefactions, we could conclude that $s$ is also constant so that $s_{L}(0-)=s_{L}^{-}, s_{R}(0+)=s_{R}^{+}$; in that case the solution is thoroughly determined. However, if we have a $j$-discontinuity, we know from the last equation in (72) that it is in fact completely determined by the $\mathbf{w}_{2 m}^{*}$ components which are already known, so that, in that case too, the argument is completed.

Note that the coupling we have performed is conservative, meaning that

$$
\mathbf{f}_{L}(\mathbf{u}(0-, t))=\mathbf{f}_{R}(\mathbf{u}(0+, t)) .
$$

Indeed, at the interface, (64) shows that $\mathbf{w}_{k}$ is conserved (the corresponding flux is null), while $\mathbf{w}_{2 m}^{*}$ being continuous, the remaining components of the left and right fluxes are equal. This also holds in conservative variables $\mathbf{u}$, since constant linear combinations of the above variables remain continuous at the interface. 
5.3. Conclusion and perspective. We have been able to make explicit coupling conditions in physical variables and then to solve the coupled Riemann problem in a unique way for a rather wide class of fluid systems whose equations are written in Lagrangian coordinates. Since the interface is characteristic, only the Riemann invariants of the eigenvalue $\lambda=0$ are constant.

Let us note by $\mathbf{W}_{L, R}\left(\xi ; \mathbf{u}_{-}, \mathbf{u}_{+}\right)$this solution of the coupled Riemann problem. It can be used as a building block for a numerical scheme. Indeed, we can define a Godunov scheme with numerical flux $\mathbf{g}_{L R}^{G o d}(\mathbf{u}, \mathbf{v})$ with (see (9) and (10)) the usual Godunov schemes in each half space

$$
\left\{\begin{array}{l}
\mathbf{g}_{L R}^{G o d}\left(\mathbf{u}_{j-1 / 2}, \mathbf{u}_{j+1 / 2}\right)=\mathbf{g}_{L}^{G o d}\left(\mathbf{u}_{j-1 / 2}, \mathbf{u}_{j+1 / 2}\right), j<0, \\
\mathbf{g}_{L R}^{\text {God }}\left(\mathbf{u}_{j-1 / 2}, \mathbf{u}_{j+1 / 2}\right)=\mathbf{g}_{R}^{\text {God }}\left(\mathbf{u}_{j-1 / 2}, \mathbf{u}_{j+1 / 2}\right), j>0,
\end{array}\right.
$$

where $\mathbf{g}_{\alpha}^{\text {God }}$ denotes the Godunov flux for $\mathbf{f}_{\alpha}$ which involves the usual (i.e. uncoupled) Riemann problems and again two fluxes at the interface $j=0$ :

$$
\left\{\begin{array}{l}
\mathbf{g}_{L R}^{\text {God- }}\left(\mathbf{u}_{-1 / 2}, \mathbf{u}_{1 / 2}\right)=\mathbf{f}_{L}\left(\mathbf{W}_{L, R}\left(0-; \mathbf{u}_{-1 / 2}, \mathbf{u}_{1 / 2}\right)\right), \\
\mathbf{g}_{L R}^{\text {God }}\left(\mathbf{u}_{-1 / 2}, \mathbf{u}_{1 / 2}\right)=\mathbf{f}_{R}\left(\mathbf{W}_{L, R}\left(0+; \mathbf{u}_{-1 / 2}, \mathbf{u}_{1 / 2}\right)\right) .
\end{array}\right.
$$

The variables $\mathbf{w}_{2 m}^{*}$ involved in these fluxes coincide. For instance, for the usual Euler system, the flux is $(-v, p, p v)^{T}$ so that the two fluxes do coincide:

$$
\mathbf{f}_{L}\left(\mathbf{W}_{L, R}\left(0-; \mathbf{u}_{-1 / 2}, \mathbf{u}_{1 / 2}\right)\right)=\mathbf{f}_{R}\left(\mathbf{W}_{L, R}\left(0+; \mathbf{u}_{-1 / 2}, \mathbf{u}_{1 / 2}\right)\right)=\left(-v_{0}, p_{0}, p_{0} v_{0}\right)^{T}
$$

if $v_{0}$ and $p_{0}$ denote the common velocity and pressure of $\mathbf{W}_{L, R}\left(0-; \mathbf{u}_{-1 / 2}, \mathbf{u}_{1 / 2}\right)$.

The above analysis can also be used for the coupling of two Euler systems (in Eulerian coordinates) in primitive variables using a Lagrange+projection scheme. In the Lagrangian step, we solve the Lagrangian system on one time step with some two-flux method which ensures the transmission of $v, p$ and then project back on the Eulerian grid, thus the Lagrangian step ensures the continuity of the Riemann invariants $\mathbf{w}_{2 m}^{*}$, i.e. of $v, p$ for the usual Euler system. A special treatment of the projection step (with mean pressure projection) will preserve this continuity. This has been performed and the corresponding scheme is used for the coupling of two Euler systems with different $\gamma$-law (we refer to [4).

This work falls within the scope of a joint research program on multiphase flows between CEA and University Pierre et Marie Curie-Paris 6 in the framework of the Neptune project (see [2, 4] for the coupling of multiphase flow models).

\section{REFERENCES}

1. Abgrall R., Karni, S., Computations of compressible multifluids. Journal of Computational Physics, 169, 594-623 (2001) MR.1836526 (2002b:76077)

2. Ambroso, A., Chalons, C., Coquel, F., Godlewski, E., Lagoutière, F., Raviart, P.-A., Seguin, N., Homogeneous models with phase transition: coupling by volume methods, in Finite Volume for Complex Applications IV, Hermes Science (2005)

3. Ambroso, A., Chalons, C., Coquel, F., Godlewski, E., Lagoutière, F., Raviart, P.-A., Couplage de deux systèmes de la dynamique des gaz. 17ème congrès Français de mécanique (sept. 2005)

4. Ambroso, A., Chalons, C., Coquel, F., Godlewski, E., Lagoutière, F., Raviart, P.-A., Seguin, N., Hérard, J.-M., Coupling of multiphase flow models. NURETH-11, International Topical Meeting on Nuclear Thermal-Hydraulics (2005)

5. Ambroso, A., Chalons, C., Coquel, F., Godlewski, E., Lagoutière, F., Raviart, P.-A., Seguin, N., Extension of interface coupling to general Lagrangian systems, Enumath 2005 proceedings, 852-860 Springer-Verlag (2006) MR2303716 
6. Chalons, C., Raviart, P.-A., Seguin, N., The interface coupling of the gas dynamics equations, to appear in Quart. Appl. Math.

7. Després, B., Lagrangian systems of conservation laws. Invariance properties of Lagrangian systems of conservation laws, approximate Riemann solvers and the entropy condition. Numer. Math., 89, Vol. 1, 99-134 (2001) MR1846766 (2002h:65122)

8. Dubois, F., Le Floch, P., Boundary conditions for nonlinear hyperbolic systems of conservation laws. J. of Diff. Equations, 71, 93-122 (1988) MR922200 (89c:35099)

9. Gisclon, M., Étude des conditions aux limites pour un système strictement hyperbolique via l'approximation parabolique. J. Math. Pures Appl., 75 (1996), 485-508 MR1411161 (97f:35129)

10. Gisclon, M., Serre, D., Conditions aux limites pour un système strictement hyperbolique fournies par le schéma de Godunov. $M^{2}$ AN, 31, (1997), 359-380 MR1451347 (98c:65152)

11. Godlewski E., Raviart, P.-A., Numerical approximation of hyperbolic systems of conservation laws, Appl. Math. Science 118, Springer, New York (1996) MR1410987 (98d:65109)

12. Godlewski E., Raviart, P.-A., The numerical interface coupling of nonlinear hyperbolic systems of conservation laws: I. The scalar case. Numer. Math., 97, 81-130, (2004) MR2045460 (2005e:65130)

13. Godlewski, E., Le Thanh, K.-C., Raviart, P.-A., The numerical interface coupling of nonlinear hyperbolic systems of conservation laws: II. The case of systems. ESAIM:M2AN, 39, 649-692 (2005) MR2165674 (2006h:65133)

14. Serre, D., Systems of conservation laws, I, II, Cambridge U. Press (2000) MR 1775057 (2001c:35146)

15. Smith, R.G., The Riemann problem in gas dynamics. Trans. Amer. Math. Society, 249, 1-50, (1979) MR 526309 (82g:35075)

DEN/DM2S/SFME, CEA-Saclay, F-91191, Gif-Sur-Yvette cedex, France

Université Paris Diderot, Laboratoire Jacques-Louis Lions, Paris, F-75005 France

Université Pierre et Marie Curie-Paris 6, UMR 7598 Laboratoire Jacques-Louis Lions, Paris, F-75005 France and CNRS, UMR 7598 Paris, F-75005 France

E-mail address: coquel@ann.jussieu.fr

Université Pierre et Marie Curie-Paris 6, UMR 7598 Laboratoire Jacques-Louis Lions, Paris, F-75005 France and CNRS, UMR 7598 Paris, F-75005 France

E-mail address: godlewski@ann.jussieu.fr

Current address: BC 187, 4 place Jussieu, 75252 Paris cedex, France

Université Paris Diderot, Laboratoire Jacques-Louis Lions, Paris, F-75005 France AND CNRS, UMR 7598 PARIS, F-75005 France

Université Pierre et Marie Curie-Paris 6, UMR 7598 Laboratoire Jacques-Louis Lions, Paris, F-75005 France and CNRS, UMR 7598 Paris, F-75005 France

Université Pierre et Marie Curie-Paris 6, UMR 7598 Laboratoire Jacques-Louis Lions, Paris, F-75005 France and CNRS, UMR 7598 Paris, F-75005 France 